\title{
BETP degradation simultaneously targets acute myelogenous leukemic stem cells and the microenvironment
}

\author{
Sujan Piya, ${ }^{1,2}$ Hong Mu, ${ }^{1,2}$ Seemana Bhattacharya, ${ }^{1,2}$ Philip L. Lorenzi, ${ }^{3}$ R. Eric Davis, ${ }^{4}$ Teresa McQueen, ${ }^{1,2}$ Vivian Ruvolo, ${ }^{1,2}$ \\ Natalia Baran, ${ }^{1,2}$ Zhiqiang Wang, ${ }^{4}$ Yimin Qian, ${ }^{5}$ Craig M. Crews, ${ }^{6}$ Marina Konopleva, ${ }^{2}$ Jo Ishizawa, ${ }^{2}$ M. James You, \\ Hagop Kantarjian, ${ }^{2}$ Michael Andreeff,, ${ }^{1,2}$ and Gautam Borthakur ${ }^{2}$
}

${ }^{1}$ Section of Molecular Hematology and Therapy, ${ }^{2}$ Department of Leukemia, ${ }^{3}$ Department of Bioinformatics and Computational Biology, and ${ }^{4}$ Department of Lymphoma and Myeloma, University of Texas MD Anderson Cancer Center, Houston, Texas, USA. ${ }^{5}$ Arvinas Inc., New Haven, Connecticut, USA. ${ }^{6}$ Department of Molecular, Cellular, and Developmental Biology, Yale University, New Haven, Connecticut, USA.

${ }^{7}$ Department of Hematopathology, University of Texas MD Anderson Cancer Center, Houston, Texas, USA

The antileukemic effect of inhibiting bromodomain and extra-terminal domain-containing (BET-containing) proteins (BETPs) such as BRD4 has largely been largely attributed to transcriptional downregulation of cellular anabolic and antiapoptotic processes, but its effect on the bone marrow microenvironment, a sanctuary favoring the persistence of leukemic stem/progenitor cells, is unexplored. Sustained degradation of BETP with the small-molecule BET proteolysistargeting chimera (PROTAC) ARV-825 resulted in a marked downregulation of surface CXCR4 and CD44, key proteins in leukemia-microenvironment interactions, in acute myeloid leukemia (AML) cells. Abrogation of surface CXCR4 expression impaired SDF-1 $\alpha$-directed migration and was mediated through transcriptional downregulation of PIM1 kinase, which in turn phosphorylates CXCR4 and facilitates its surface localization. Downregulation of CD44, including isoforms CD44v8-10 impaired cystine uptake, lowered intracellular reduced glutathione, and increased oxidative stress. More important, BETP degradation markedly decreased the CD34+CD38-CD90-CD45RA+ leukemic stem cell population and, alone or in combination with cytarabine, prolonged survival in a mouse model of human leukemia that included AML patient-derived xenografts (AML-PDX). Gene expression profiling and single-cell proteomics confirmed a downregulation of the gene signatures associated with "stemness" in AML and Wnt/ $\beta$-catenin and Myc pathways. Hence, BETP degradation by ARV-825 simultaneously targets cell-intrinsic signaling, stromal interactions, and metabolism in AML.

\section{Introduction}

Acute myeloid leukemia (AML) is the most common hematological malignancy in adults and the second most common one in children (1). Aberrant epigenetic events associated with DNA/ chromatin modification that enhance oncogene expression and/ or block differentiation are central to AML pathogenesis (2-4). Unbiased shRNA-based and genome-wide CRISPR-based screening for epigenetic targets for therapy in AML resulted in the identification of BETP, the most important member of the BET family of histone reader proteins, as critical for AML maintenance $(5,6)$. BETP binds to acetylated lysine residues in histone $\mathrm{H} 4$ and provides the scaffold to assemble multimolecular super-enhancer complexes that drive the expression of oncogenes $(7,8) M Y C$ is one of the key oncogenes that depend on BETP-containing superenhancer complex-mediated transcription (9-11) and orchestrate a potent transcription program encompassing multiple oncogenic

Conflict of interest: The authors have declared that no conflict of interest exists. Copyright: () 2019, American Society for Clinical Investigation.

Submitted: February 22, 2018; Accepted: February 12, 2019; Published: March 25, 2019. Reference information: J Clin Invest. 2019;129(5):1878-1894. https://doi.org/10.1172/JCl120654. pathways. Myc, as a transcription factor, is challenging to target directly, given the lack of pockets that could be targeted directly with small molecules, and hence much effort has been focused on indirect targeting strategies. Prototypic small-molecule bromodomain-containing 4 (BRD4) inhibitors like JQ1 and I-BET, which disrupt BETP binding to acetylated histones $(7,12,13)$, have provided a means to target MYC transcriptionally by way of disrupting "super-enhancer" transcription complexes. However, these agents have limited clinical activity and a general lack of ability for sustained transcriptional inhibition of targets (14). Mechanisms implicated in resistance to BRD4 inhibitors include increased BRD4 expression, BRD4-independent oncogene expression, and/ or alternative transcriptional pathways that drive Myc expression (e.g., Wnt/ $\beta$-catenin signaling) in leukemic stem cells (LSCs) (1518). In that context, BRD4 and BET degraders provide a unique opportunity to profoundly impair BRD4-driven transcription and overcome resistance mechanisms that are still dependent on BRD4-based transcription. ARV-825 is a proteolysis-targeting chimera (PROTAC) that targets BRD4 and BET family proteins for cereblon-mediated proteasomal degradation and sustained downregulation of BRD4-dependent transcriptional function. Saenz et al. demonstrated that ARV- 825 mediates BETP degradation and is 

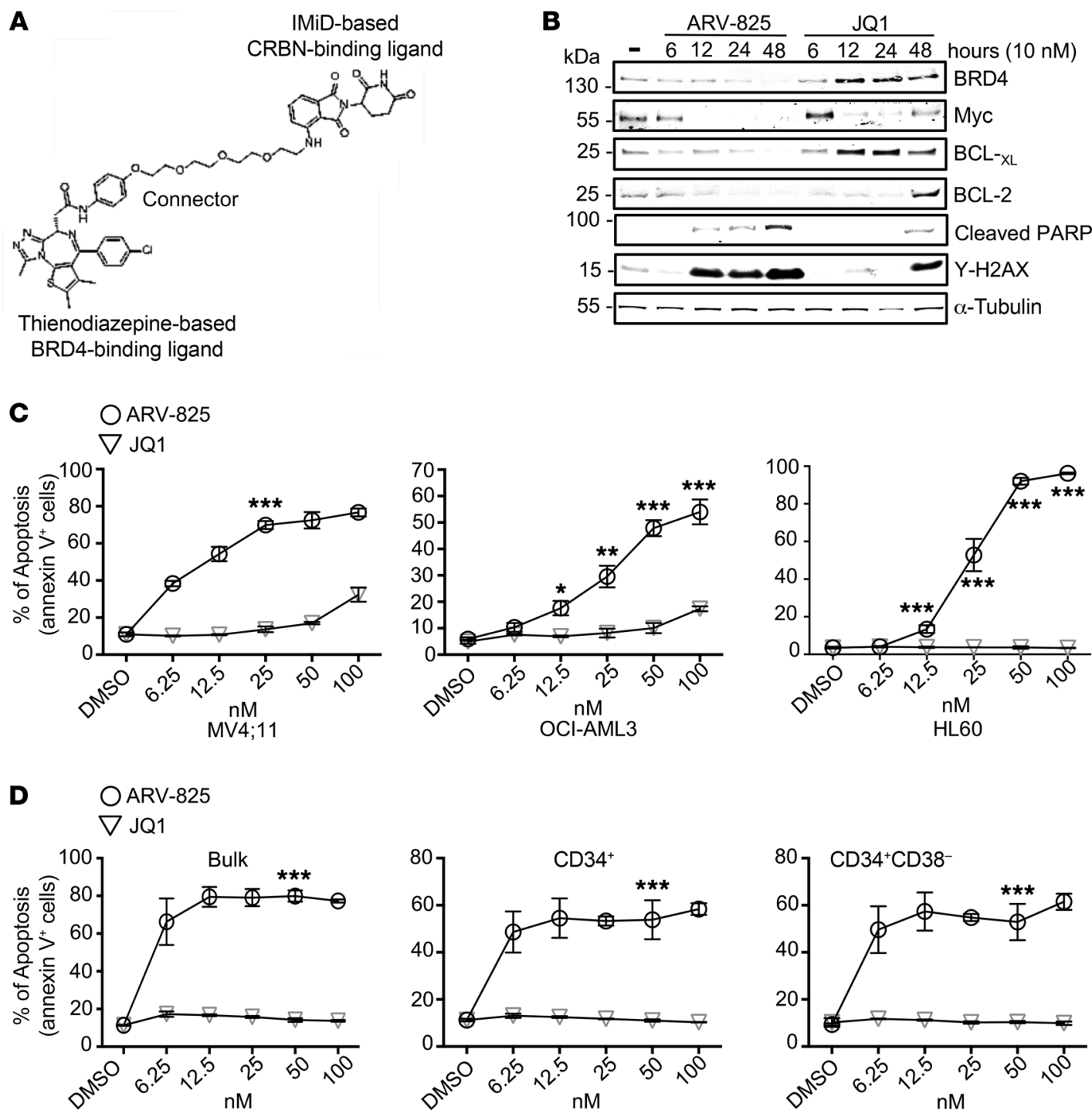

Figure 1. ARV-825 causes lasting degradation of BRD4 and is a more potent inhibitor of cell proliferation and apoptosis in patient-derived AML or stem/progenitor cells than are small-molecule BET inhibitors. (A) Chemical structure of ARV-825. (B) OCI-AM3 cells were treated with ARV-825 or JQ1 (10 $\mathrm{nM}$ ) for different durations. Whole-cell lysates were resolved on SDS-PAGE, transferred to PVDF membranes, and probed with the indicated antibodies. $\alpha$-Tubulin was used as a loading control. (C) AML cell lines were treated for 72 hours with ARV- 825 or JQ1 in a wide range of concentrations and then stained with annexin $V$ and DAPI to measure the percentage of apoptosis in cells. (D) Primary AML blasts were cultured with increasing concentrations of ARV-825 or JQ1. After 72 hours, cell pellets were stained with annexin V, CD45, CD34, and CD38, and cell survival in different subpopulations was determined by flow cytometry. Data represent the mean \pm SD from 3 independent experiments. The statistical significance between 2 groups was calculated using a standard Student's $t$ test $\left({ }^{*} P<0.05,{ }^{* *} P<0.01\right.$, and $\left.{ }^{* * *} P<0.001\right)$.

more effective than existing BET inhibitors at blocking BETP transcriptional function in myeloproliferative neoplasms (MPNs) (19). The MPN phenotype is less aggressive than AML, and the core mutation profile is different from that of AML, except in advanced disease, prompting us to study BRD4/BET degradation in AML with an additional focus on the microenvironment.

The leukemia bone marrow (BM) microenvironment plays a central role in the protection of LSCs and persistence of residual disease in AML (20). LSC-relevant stromal interactions include those involving chemokines and adhesion molecule/ligand signaling by SDF1- $\alpha / C X C R 4$, CD44 and its variants (CD44v/hyaluronic acid), osteopontin, etc. (21-23). CD44v8-10, a variant transcript of CD44, is implicated in the maintenance of leukemia-initiating cells in acute leukemias $(24,25)$. Functionally, CD44v8-10 stabilizes ${ }_{X} \mathrm{CT}$, a subunit of the cystine-glutamate transporter ${ }_{\mathrm{X}} \mathrm{C}(-)$, which promotes cystine uptake for glutathione (GSH) synthesis and mitigation of intracellular oxidative stress $(26,27)$. Quiescent LSCs depend on a low ROS state for their maintenance (28). To successfully translate BRD4/BET degradation strategies for AML therapy, particularly in the elimination of LSCs, it is critical to define the effect of BRD4/BET on the leukemia microenvironment.

We used the PROTAC ARV-825 to achieve sustained depletion of BRD4 and BET proteins (16). ARV-825 exhibited robust antileukemic activity and markedly reduced BETP-dependent transcription of oncogenes, antiapoptotic proteins, and chemokine 
receptors. ARV-825 was 10- to 100-fold more potent against AML cells (including primary samples) than were prototypic BRD4 inhibitors such as JQ1. ARV-825 was active against $\mathrm{CD} 34^{+} \mathrm{CD} 38^{-}$ putative leukemia progenitor cells but spared healthy BM-derived progenitor cells. Time-of-flight mass spectrometry-based flow cytometry (CyTOF) analysis (including AML patient-derived xenografts [PDX] cells) revealed that ARV-825 not only downregulated prosurvival proteins (Myc, B cell leukemia/lymphoma 2 [BCL-2], myeloid cell leukemia sequence 1 [MCL-1], etc.), but also profoundly suppressed surface expression of CXCR4 (but not total CXCR4) and CD44 in the LSC compartment. Additionally, ARV-825 reduced intracellular cystine, increased cellular ROS, and downregulated the expression of genes associated with the LSC signature and the Wnt/ $\beta$-catenin pathway. Finally, ARV-825 reduced LSC burden and improved survival in a mouse model of disseminated AML (including PDX), indicating its potential for clinical development.

\section{Results}

ARV-825 inhibits AML cell proliferation and induces apoptosis. ARV-825, the heterobifunctional small-molecule degrader of $\mathrm{BET}$ proteins, contains a ligand for a BET protein connected via a linker to a ligand for the E3 ubiquitin ligase cereblon (Figure 1A). ARV-825 treatment resulted in sustained degradation of BRD4 and downregulation of its transcriptional targets Myc, BCL-2, and $\mathrm{BCL}_{\mathrm{XL}}$ (Figure $1 \mathrm{~B}$ ), accompanied by poly-(ADP-ribose) polymerase (PARP) cleavage and an increase in $\mathrm{Y}-\mathrm{H} 2 \mathrm{AX}$. In the same assay, JQ1 treatment led to a less sustained downregulation of transcriptional targets and an increase in BRD4 protein levels (Figure 1B). ARV-825 inhibited AML cell proliferation in the low-picomolar to low-nanomolar range, with $\mathrm{IC}_{50}$ values that were 10- to 1000-fold lower than those for JQ1 (Supplemental Table 1; supplemental material available online with this article; https://doi.org/10.1172/JCI120654DS1). The more potent activity of ARV-825 corresponded to an enhanced apoptotic response in MV4-11, MOLM-13, and OCI-AML3 cells, as measured by annexin $\mathrm{V}$ staining (Figure 1C), and inhibition of cell proliferation, as indicated by a decrease in absolute cell numbers (Supplemental Figure 1A).

We further analyzed the effects of ARV-825 or JQ1 on primary AML cells carrying mutations relevant to AML including mutations in FLT3 (residue D835) (Figure 1D), ASXL, TP53, MPL (Supplemental Figure 1B), JAK2, MPL, WT1, CEBPA (Supplemental Figure 1C), KIT, IDH2 (Supplemental Figure 1D), NPM1, RUNX1, IDH2, FLT-ITD (Supplemental Figure 1E), NPM1, and IDH2 (Supplemental Figure 1F). ARV-825 demonstrated higher potency than JQ1 in inducing apoptosis in bulk and, more important, in $\mathrm{CD}^{+} 4^{+}$and $\mathrm{CD} 34^{+} \mathrm{CD} 38^{-} \mathrm{AML}$ stem/progenitor cells. At relevant doses, ARV-825 was nontoxic to hematopoietic progenitor cells from normal (healthy) human donor-derived BM samples (Supplemental Figure $1 G)$.

$A R V-825$ induces sustained downregulation of $c-M y c$. Given its mechanism of action, once ARV-825 enters the cell, it should be available for repeated and sustained degradation of BET proteins, even after withdrawal of the agent from the culture medium. To test this hypothesis, we removed ARV-825 from growth media after a 24-hour exposure and continued culturing using normal growth media. As expected, inhibited cell proliferation, apoptosis (Supplemental Table 2), BRD4 degradation, and inhibited Myc expression were sustained for at least 48 hours after washout (Figure 2, A and B). After washout of JQ1, however, BRD4 and Myc levels rebounded (Figure 2B), and inhibition of cell proliferation (Figure 2A) and induction of apoptosis (Figure 2A) were abrogated. We observed similar results in a primary AML sample (Figure 2C).

Myc is considered a key transcriptional target of BRD4mediated transcription, and we found that ectopic Myc expression partially rescued ARV-825-induced cell death, as indicated by the decrease in apoptosis and cleaved PARP in Myc-overexpressed cells (Figure 2, D and E). Since transcription of BCL-2 and BCLis also BRD4 mediated, we found that BCL-2 and $\mathrm{BCL}^{-}{ }_{\mathrm{XL}}$ overexpression at least partially abrogated ARV-825-induced cell death (Supplemental Figure 2).

$A R V-825$ overcomes microenvironment-mediated drug resistance and alters PIM1-mediated phosphorylation and surface expression of CXCR4. Leukemic stem/progenitor cells reside in the BM niche, which provides the structure and physiologic conditions to activate prosurvival signals, overcome nutrient limitations, and adapt to hypoxia, all of which are mechanisms associated with evasion of chemotherapy-induced cell death $(29,30)$. To better understand the efficacy of ARV-825 in the AML microenvironment, we cocultured OCI-AML3 cells with normal (healthy) donor BM-derived mesenchymal stromal cells (NMSCs) and treated them with ARV$825(50 \mathrm{nM})$ or cytarabine $(1 \mu \mathrm{M})$ under normoxic or hypoxic conditions. Coculture with MSCs rendered OCI-AML3 cells relatively resistant to cytarabine under both conditions. Conversely, sensitivity to ARV-825 was the same in mono- or coculture and under both $\mathrm{O}_{2}$ conditions (Figure $3 \mathrm{~A}$ ), suggesting that ARV-825 could overcome stroma- and hypoxia-mediated resistance.

To gain insight into the microenvironmental effects, we performed CyTOF to simultaneously profile multiple surface and intracellular molecules involved in leukemia/stroma interactions and apoptosis. In OCI-AML3 cells, in addition to known BRD4 targets like Myc, BCL-2, BCL- ${ }_{\mathrm{XL}}$, and MCL-1, treatment with ARV-825 substantially decreased the expression of 2 key molecules involved in leukemia/stroma interaction: surface CXCR4 (but not total CXCR4) and total CD44 (Figure 3B). Using conventional flow cytometry (Figure $3 \mathrm{C}$ ) and confocal imaging (Figure 3D), with and without permeabilization of AML cells, we confirmed that ARV-825 downregulated only surface CXCR4 expression. These data suggested that decreased surface expression of CXCR4 is not driven by impaired CXCR4 transcription resulting from BETP inhibition.

The CXCR4/SDF- $1 \alpha$ signaling axis is important in leukemic cell homing, migration, and survival via activation of ERK and PI3K $(31,32)$. As a potential functional correlate of cell-surface CXCR4 expression, we examined the chemotaxis of OCI-AML3 and primary AML cells treated with ARV- 825 toward a SDF- $1 \alpha$ gradient. As expected, reduced surface expression of CXCR4 with ARV-825 treatment correlated with a 3 -fold decrease in the migration of AML cells (OCI-AML3 and primary AML) toward SDF- $1 \alpha$ (Figure 3E and Supplemental Figure 3A). The decreased chemotaxis was associated with dephosphorylation of ERK (Figure 3E) in the presence of SDF-1, which indicated abrogation of stromal signaling. 

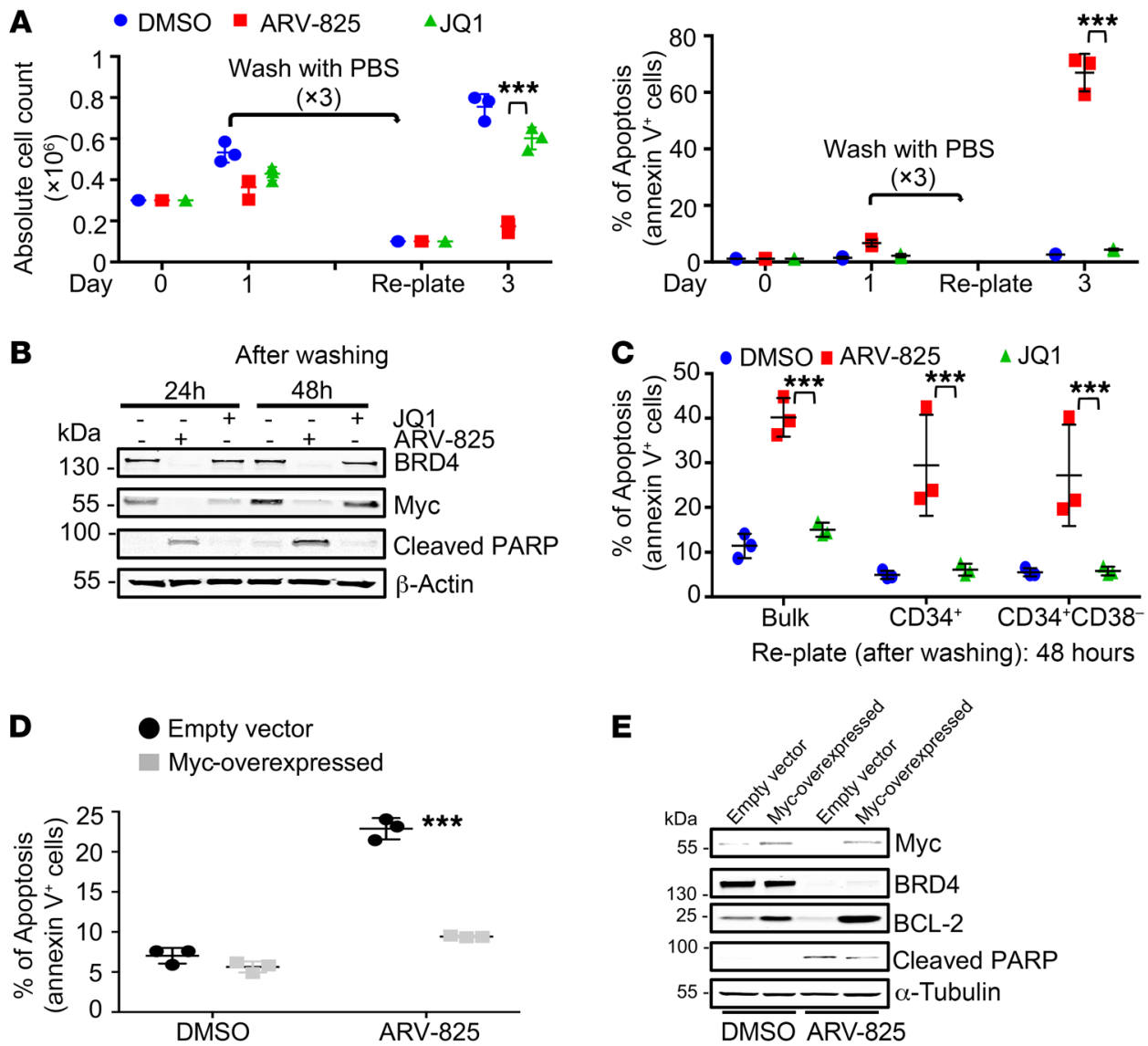

Figure 2. ARV-825 exerts a sustained effect compared with JQ1. OCI-AML3 cells were treated with ARV-825 (10 nM), JQ1 (100 nM), or DMSO for 24 hours. Cells were washed with PBS to remove the respective drugs and re-plated in equal numbers in complete media without any drug for an additional 48 hours. After 48 hours, the apoptosis percentage and cell numbers were assessed, and lysates were used for immunoblotting. (A) Absolute cell numbers and apoptosis percentage were measured by Beckman Vi-CELL counts and flow cytometry, respectively $(n=3)$. (B) Immunoblot analysis to detect the indicated proteins. $\beta$-Actin was used as a loading control. (C) Primary AML cells (sample 2) were treated with DMSO, ARV-825 (50 nM), or JQ1 (100 nM) for 24 hours, washed 3 times with PBS to remove traces of the drug, and re-plated in equal numbers for another 48 hours. Cells were then subjected to annexin V, CD45, CD34, and CD38 staining to measure the percentage of apoptosis from 3 independent samples. ( $\mathbf{D}$ and $\mathbf{E}$ ) The transient Myc-overexpressed and empty vector control OCI-AML3 cells were treated with ARV-825 (10 nM) for 72 hours or 24 hours and then subjected to an apoptosis assay from 3 independent samples or to immunoblotting to detect the different protein expression levels, respectively. The statistical significance between 2 groups was calculated using a standard Student's $t$ test $\left({ }^{* *} P \leq 0.001\right)$.

Apart from transcription and protein translation, cell-surface expression of CXCR4 depends on its recycling and externalization on the cell membrane (33). PIM1-mediated phosphorylation of CXCR4 at S339 is necessary for its surface localization (34-36), and PIM1 is a known downstream target of BETP (37). Immunoblot analysis of OCI-AML3 cells treated with ARV-825 confirmed that PIM1 expression and the consequent phosphorylation of CXCR4 (S339) were decreased in the treated cells, while total CXCR4 protein expression remained unchanged (Figure $3 \mathrm{~F}$ ). These data suggest that ARV-825 alters PIM1 kinase-mediated surface CXCR4 expression and not total CXCR4.

To experimentally address this possibility, we overexpressed PIM1 in OCI-AML3 cells and measured the cell-surface expression of CXCR4 as well as SDF-1-directed chemotaxis. As shown in Figure 3G, overexpression of PIM1 partially reversed the loss of phosphorylated CXCR4 (p-CXCR4) in AML cells treated with ARV-825, resulting in a partial rescue of CXCR4 surface expression (Figure $3 \mathrm{H}$ ) and restoration of AML cell migration toward
SDF-1 $\alpha$ (Figure 3H). These results strongly suggest that ARV-825 regulates the PIM1-mediated phosphorylation of CXCR4 that is necessary for its externalization.

Anticipating the potential pan-BET family effect of ARV-825, we measured expression levels of BRD2, BRD3, and BRD4, Myc, PIM1, and p-CXCR4 along with expression of the BRD4-specific degrader MZ1. Indeed, at relevant $\mathrm{IC}_{50}$ concentrations, ARV-825 showed preferential BRD4 degradation compared with MZ1, with decreased p-CXCR4 (Supplemental Figure 3, B and C) and a similar phenotype of reduced surface expression of CXCR4 (Supplemental Figure 3D).

ARV-825 downregulates CD44v8-10, increases ROS levels, and suppresses oxidative phosphorylation in AML cells. CD44 and its variants have been implicated as regulators of amino acid transport and cellular redox balance, particularly through cystine import $(38,39)$. SLC7A11, a cystine-glutamate antiporter, is stabilized in the plasma membrane by $\mathrm{CD} 44 \mathrm{v} 8-10$ through its interaction with and stabilization of ${ }_{\mathrm{X}} \mathrm{CT} / \mathrm{SLC7A11}$ (27), while expression of 
A - Monoculture - Coculture

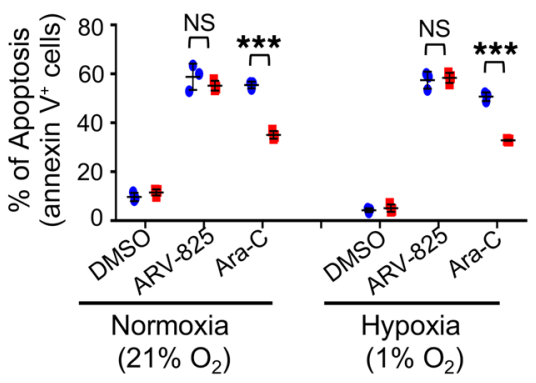

B

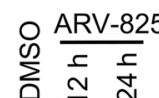

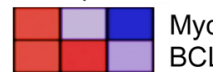

BCL- $x$ L

P21

Caspase 3

BIM

Bax

MCL-1

CXCR4 (surface)

HIF-1 $\alpha$

CD44

CD86

CD134

P53

ARC

IL-6

p-SYK

Hes1

p-AKT

p-ERK

p-STAT5

p-JNK

P38

p-AMPK

LKB1

Color scheme legend

$\begin{array}{lllll}0.2 & 0.6 & 0.8 & 1.2 & 1.4\end{array}$
C $\square$ DMSO $\square$ ARV-825 $\square$ JQ1
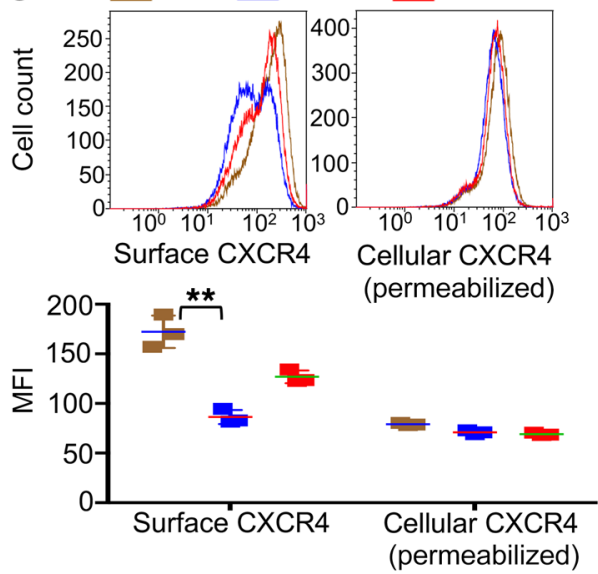

D
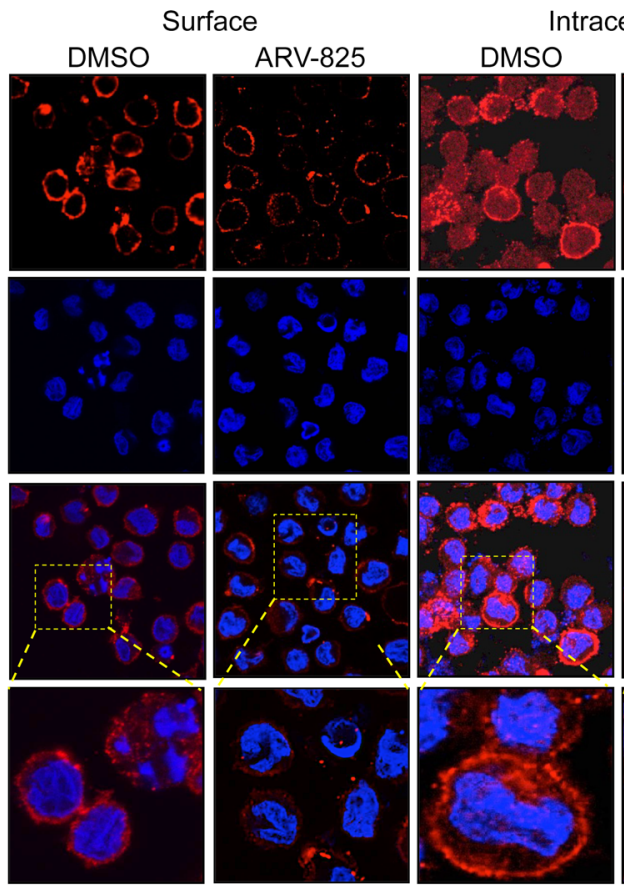

Intracellular
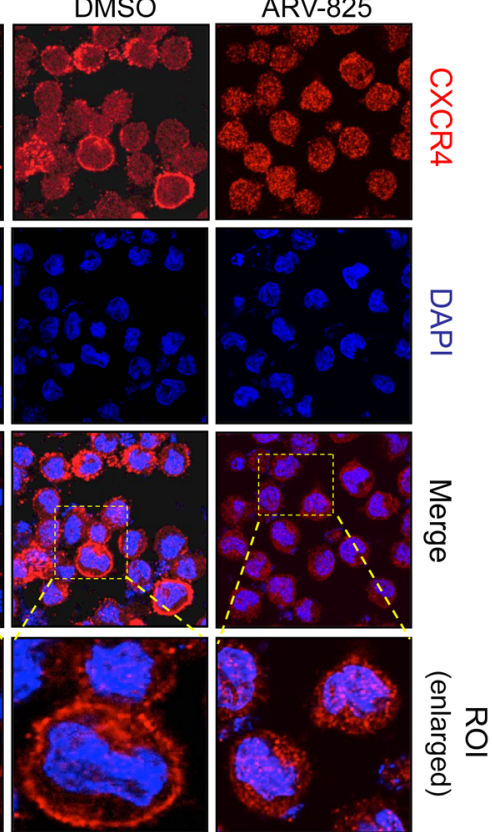

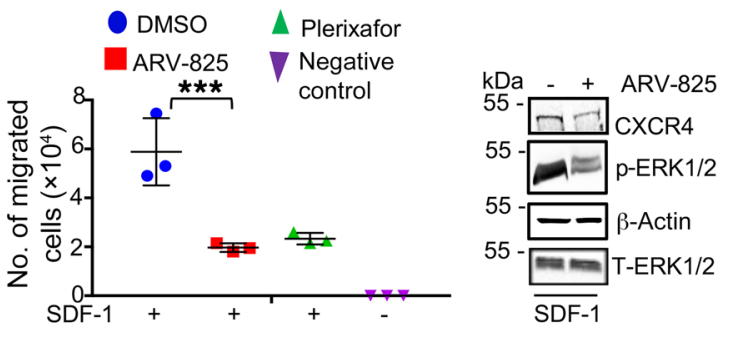

H
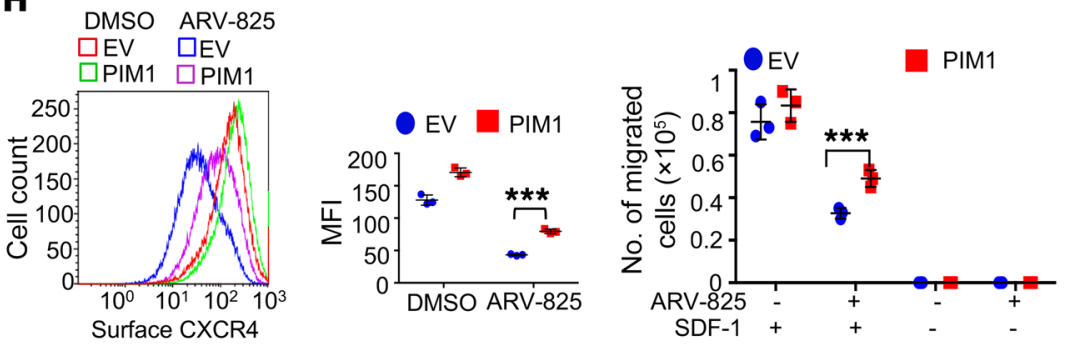

F

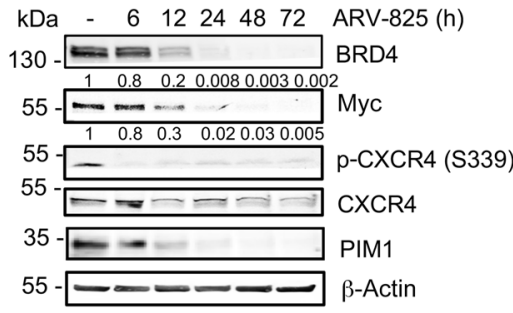

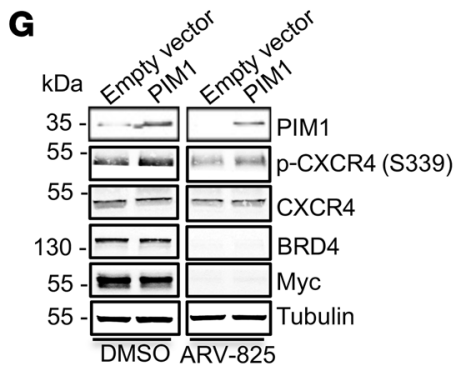


Figure 3. ARV-825 downregulates the chemolkine and adhesion receptors in leukemic cells and disrupts stromal-leukemic cell interactions to inhibit BM microenvironment niche-mediated drug resistance. (A) OCI-AML3 (normal or hypoxia-adapted) cells were cultured with or without a monolayer of NMSCs and treated with ARV-825 (50 nM) or cytarabine $(1 \mu \mathrm{M})$ under either normoxic or hypoxic conditions for 72 hours, and apoptosis was assessed by annexin $V$ assay using flow cytometry $(n=3)$. (B) OCI-AML3 cells were treated with ARV-825 ( $10 \mathrm{nM}$ ) for 12 or 24 hours and subjected to CyTOF, and a heatmap was generated using the publicly available Broad Institute Gene Pattern Heatmap viewer. OCI-AML3 cells treated for 24 hours were stained for CXCR4 with or without permeabilization to quantify the changes in intracellular or surface CXCR4 expression using (C) a PE-conjugated antibody for the flow-based assay $(n=3)$ and (D) a AF594-conjugated secondary antibody for confocal imaging (original magnification, $\times 40$ ). (E) OCI-AML3 cells were treated with ARV-825 (10 $\mathrm{nM}$ ) or plerixafor (100 nM) (positive control) for 24 hours and subjected to a migration assay 4 hours after incubation in media containing SDF-1 (100 ng). Surface expression of CXCR4 was measured by flow cytometry, and cell migration was measured by collecting the cells from the lower chamber containing SDF-1 following Beckman Vi-CELL counts ( $n=$ 3). Whole-cell lysates obtained from OCI-AML3 cells treated with or without ARV-825 in the presence of SDF-1 were processed for immunoblotting with the indicated antibodies. $\beta$-Actin served as a loading control. (F) OCI-AML3 cells were treated with ARV-825 $(10 \mathrm{nM})$ for the indicated durations, and wholecell lysates were analyzed with the indicated antibodies ( $\beta$-actin served as a loading control). Numbers indicate normalized intensity. PIM1-overexpressing OCI-AML3 cells were treated with ARV-825 $(10 \mathrm{nM})$ for 24 hours, and then (C) whole-cell lysates were analyzed by immunoblotting with the indicated antibodies ( $\alpha$-tubulin was used as a loading control). (H) Surface expression of CXCR4 was analyzed by flow cytometry followed by a migration assay $(n=3)$. ${ }^{* *} P<0.01$ and ${ }^{* *} P<0.001$, by standard Student's $t$ test.

the antiporter is induced directly by c-Myc. Quantitative real-time PCR (qPCR) and immunoblotting confirmed the CyTOF finding of pronounced downregulation of CD44 and CD44v8-10 mRNA in AML cells upon BRD4 degradation (Figure $4 \mathrm{~A}$ ). As a functional correlate of CD44v8-10 downregulation, mass spectrometric analysis showed decreased intracellular cysteine levels following treatment with ARV-825 (Figure 4B), while other intracellular amino acids remained mostly unchanged (Supplemental Figure 4). Decreased cysteine uptake coincided with decreased intracellular GSH levels (Figure 4B) and increased generation of ROS (Figure 4C). To further confirm whether elevated ROS contributes to apoptosis, we exposed AML cells treated with ARV-825 to the exogenous ROS scavenger $N$-acetyl-L-cysteine (NAC). The abrogation of ROS generation substantially reduced apoptosis, indicating that ARV-825-mediated oxidative injury contributes to ARV antileukemic activity (Figure 4D).

Antileukemic activity of ARV-825 in a xenograft mouse model of human leukemia. To test the potential of ARV-825 as single-agent AML therapy, mice transplanted with OCI-AML3-GFP-Luc leukemic cells were treated with either ARV-825 (10 mg/kg twice a week, i.p.) or vehicle. A reduction of disease burden with ARV-825 was confirmed by bioluminescence imaging (Supplemental Figure 5A). Flow cytometric analysis of peripheral blood on day 29 showed substantial clearance of human $\mathrm{CD} 45^{+}\left(\mathrm{hCD} 45^{+}\right)$cells in the ARV-825-treated mice (Supplemental Figure 5B). Consistent with the antileukemic activity of ARV-825, mice treated with this agent had a smaller spleen size (Supplemental Figure 5B), a lower leukemic cell burden (Supplemental Figure 5B), and hCD $45^{+}$infiltrates in the spleen, liver, and BM (Figure 5A, left), as measured by CD45 IHC quantitative imaging (mean $\mathrm{H}$ score for vehicle vs. ARV-825 in BM: 131.5 vs. $24.9, P \leq 0.0001$; spleen: 203.61 vs. 55.2,
$P \leq 0.0001$; and liver: 115.3 vs. $20.2, P \leq 0.0001)$, where, on the basis of Bonferroni's correction for type I errors, a $P$ value of 0.017 or less was considered statistically significant (Figure 5A, right). Target inhibition was confirmed with the reduced expression of Myc in those tissues (Supplemental Figure 5C). Interestingly, as seen in Supplemental Figure 5D, cells flushed from the BM of ARV825-treated animals showed lower surface expression of CXCR4, an observation consistent with the in vitro findings. Finally, in this aggressive AML model (Figure 5B), mice in the ARV-825 treatment cohort had significantly improved survival compared with vehicle-treated mice, suggesting that ARV-825 has single-agent efficacy against AML.

Similar experiments using an AML-PDX model (relapsed fms-related tyrosine kinase 3-internal tandem duplication [FLT3ITD], complex cytogenetics) confirmed the in vivo antileukemic effects of ARV-825, as exhibited by a reduced percentage of $\mathrm{CD} 45^{+}$cells in peripheral blood (Supplemental Figure 5E), while target inhibition was confirmed by a reduction of $\mathrm{BRD} 4$ protein expression and lower surface expression of CXCR4 and CD44 on day 75, findings that were consistent with the in vitro data (Supplemental Figure 5F). Confirming the antileukemic activity of ARV825, mice treated with ARV-825 had normal-sized spleens, and flow cytometric analysis of flushed BM cells showed lower hCD $45^{+}$ expression in $\mathrm{BM}$ and reduced leukemic cell infiltration into the spleen, liver, and BM (Figure 5, C and D). Overall, the ARV-825treated cohort had significantly improved survival compared with vehicle-treated mice (Figure $5 \mathrm{E}$ ). We observed no significant weight loss as a result of ARV-825 treatment in mice in either model over the duration of the experiment (data not shown).

$A R V-825$ has a stronger antileukemic effect than JQ1 and is synergistic with standard cytarabine therapy. To determine the merit of pursuing clinical development of ARV-825 in a relevant context, we tested the efficacy of ARV-825 alone or in combination with cytarabine and also compared it with JQ1 in an in vivo mouse model. Following the engraftment of OCI-AML3-GFP-Luc leukemic cells into sublethally irradiated NOD/SCID/IL-2r $\gamma$-null (NSG) mice, we compared the antileukemic effects of treatment with ARV-825 (10 mg/kg), JQ1 (50 mg/kg), Ara-C (50 mg/kg) and a combination of ARV-825, Ara-C, and vehicle (administered i.p. twice weekly). Consistent with the in vitro data, when compared with JQ1 (Figure 1) and the synergistic effect with Ara-C (Supplemental Figure 6A), we found that ARV-825 had a greater effect than JQ1 and that it was synergistic with Ara-C in reducing the leukemia burden, as exhibited by bioluminescence imaging (Supplemental Figure 6B), hCD45 levels in peripheral blood (Figure 6A), and reduced leukemic cell infiltration into the spleen, liver, and $\mathrm{BM}$ on day 35 (Figure 6, B and C). Of clinical relevance is the finding that ARV-825, in combination with cytarabine, showed significantly improved survival (median survival of 59 days) (Figure 6D). Animals in all treated cohorts maintained their weight until the last dose of the drug (Supplemental Figure 6C).

$A R V-825$ downregulates the Wnt/ $\beta$-catenin pathway along with c-Myc transcriptional programs in AML LSCs and key signaling programs in stromal cells. We used genome-wide gene expression profiling (GEP) to examine the transcriptional changes after treatment of OCI-AML 3 cells and a primary AML sample with ARV-825 for 24 hours. We found that ARV-825 exposure prompted a dramatic 

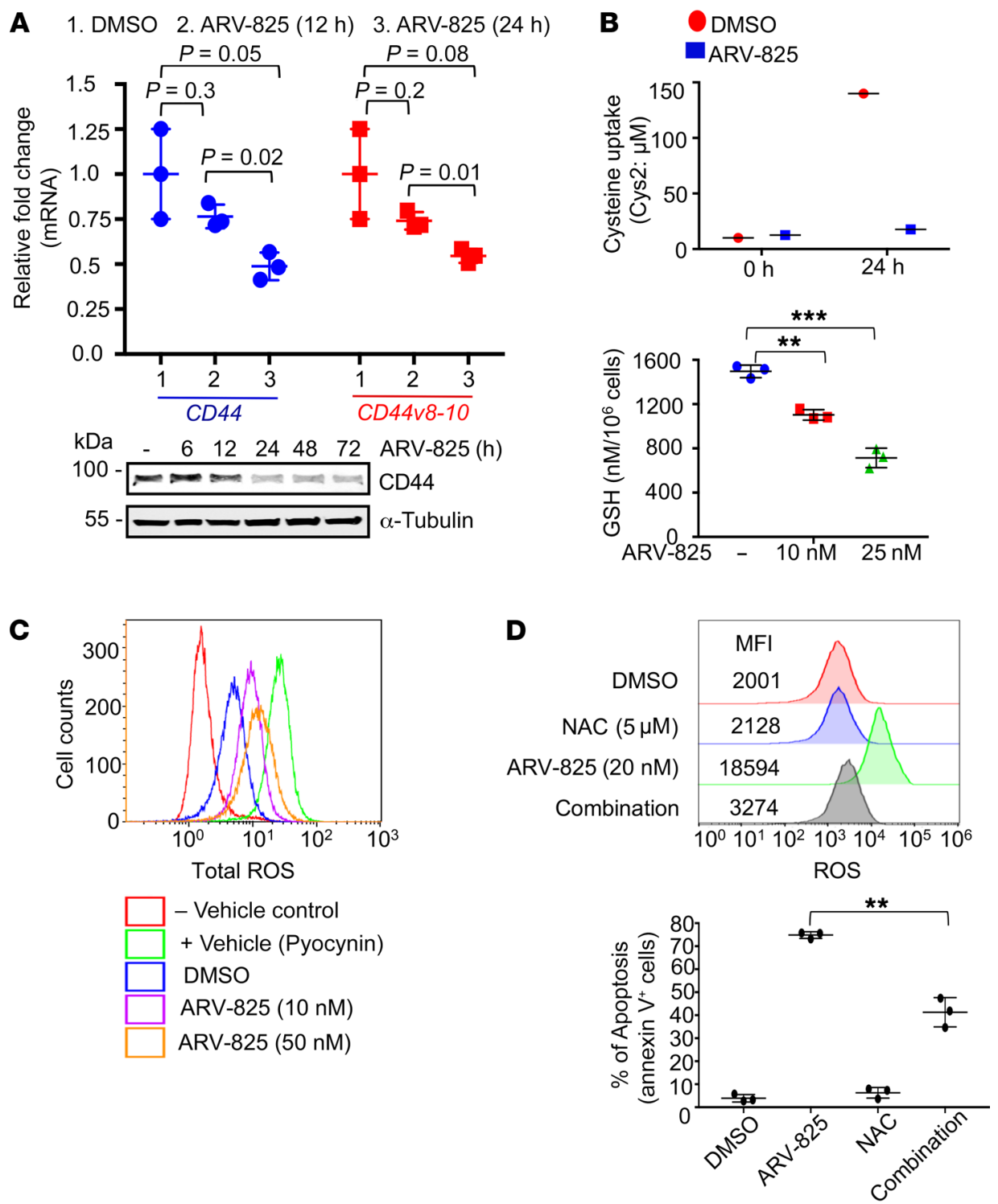

\begin{abstract}
Figure 4. ARV-825 downregulates CD44v8-10, increases ROS, and maintains oxidative stress in AML. (A) OCI-AML3 cells were cultured with ARV-825 (10 nM) for the indicated durations. RNA was extracted and qPCR performed for CD44 and CD44v8-10. Gene expression was normalized to the corresponding 185 rRNA expression level $(n=3)$. Total cell lysates were subjected to immunoblotting with CD44, and $\alpha$-tubulin served as a loading control. Statistical significance was calculated using Bonferroni's method, and the adjusted $P$ values were determined. (B) OCI-AML 3 cells were treated with ARV-825 (10 nM) for 24 hours, and mass spectrometry-based analysis was performed to assess cysteine uptake. Cys2, intracellular cysteine considered as cysteine uptake. Analysis of the $\mathrm{GSH}$ content was done using a Cayman Chemical kit ( $n=3$ independent samples). (C) Duplicate samples were subjected to an assay to determine total ROS generation using an ENZ-51011 kit. (D) OCI-AML3 cells were preincubated with NAC for 1 hour, followed by ARV$825(10 \mathrm{nM})$ treatment for 24 hours and a 72-hour incubation for ROS detection and apoptosis assay, respectively $(n=3)$. ${ }^{* *} P<0.01$ and ${ }^{* * *} P<0.001$, by standard Student's $t$ test.
\end{abstract}

change in the transcriptome: 1006 downregulated genes and 786 upregulated genes met the significance criteria $(P \leq 0.01)$ for $\mathrm{a} \geq 1$ change in the $\log _{2}$ value (Supplemental Figure 7A), and many genes showed much larger fold changes. We performed gene set enrichment analysis (GSEA) using gene signatures from the Molecular Signatures Database (MSigDB). There was significant enrichment indicating downregulation of Myc target genes along with gene sets representing other oncogenic pathways, cell-cycle progression, the hypoxia response, metabolism, and Notch pathway activity (Figure 7A). Specifically, GSEA revealed a marked downregulation of Myc-specific and Wnt/ $\beta$-catenin pathways with ARV-825 treatment (Supplemental Figure 7A). This was validated by qPCR analysis of the Wnt/ $\beta$-catenin pathway target genes AXIN2 and FRA1 (Figure 7A), whose mRNA levels were significantly downregulated in OCI-AM3 cells after 12 and 24 hours of ARV-825 treatment. These findings may have significant clinical implications, since Wnt/ $\beta$-catenin-driven Myc transcription has been identified as a resistance pathway for LSC populations in response to BET inhibition with small-molecule BRD4 inhibitors like JQ1 and I-BET (15).
Since stromal cells in the BM provide a sanctuary, by integrating signaling pathways including SDF1/CXCL12, Wnt/ $\beta$-catenin, VCAM/VLA-4/NF-кB，CD44，PI3K/AKT/mTOR, and hypoxia, that contributes to LSC survival $(24,31)$, we used genome-wide GEP to examine the effect of 24 hours of ARV-825 treatment on NMSCs. We found that, compared with AML cells, ARV-825 exposure induced a relatively smaller change in the transcriptome: 340 downregulated genes and 140 upregulated genes met the significance criteria $(P \leq 0.01)$ for a $\geq 1$ change in the $\log _{2}$ value (Supplemental Figure 7B). GSEA revealed downregulated genes in pathways related to protein secretion, Myc, Wnt/ $\beta$-catenin, Notch, and PI3K/AKT/mTOR (Figure 7B). From a microenvironment perspective, GSEA showed a reduction in hyaluronan synthase 2 (HAS2), vascular cell adhesion protein 1 (VCAM-1), and C-X-C motif chemokine 12 (CXCL12, also known as SDF-1), molecules that engage with CD44, VLA4, and CXCR4, respectively, on AML cells. In contrast to AML cells, expression of solute carrier family 7 member 11 (SLC7A11) and cystine transporter ${ }_{\mathrm{X}} \mathrm{CT}$ was upregulated in NMSCs (Figure 7B). Immu- 
noblotting confirmed BRD4 and Myc downregulation, but unlike AML cells, we observed no changes in CD44 or ${ }_{\mathrm{X}} \mathrm{CT}$ expression (Supplemental Figure 7C). BM stromal cells effectively import cystine and convert it to cysteine, which is then released into the microenvironment for uptake of leukemic cells to promote GSH synthesis, and this leukemic-stromal cell interaction is critical for cell survival (40). Therefore, we sought to ascertain whether coculture with stromal cells would allow leukemic cells to abrogate the ROS effect by shuttling cysteine to leukemic cells. In AML and stromal cell coculture experiments, however, we found that ARV-825 did not allow the rescue of AML cells from ${ }_{\mathrm{x}} \mathrm{CT}$ inhibition (Figure $7 \mathrm{C}$ ) or the abrogation of increased ROS in AML cells (Figure 7C).

A recent study by $\mathrm{Ng}$ et al. (41) fractionated samples from AML patients according to expression of CD34 and/or CD38 and functionally assigned fractions to LSC or non-LSC categories by xenotransplantation. We then determined the gene expression of these fractions and developed a 17-gene signature score for AML outcomes. Next, we performed GSEA of publicly available GEP data (Gene Expression Omnibus [GEO] GSE76009) and found that Myc targets were highly expressed in LSC fractions (Figure 7D). However, a gene array of ARV-825-treated primary AML cells with complex cytogenetics and FLT3-ITD mutation showed reduced Myc activity as well as downregulation of a majority of the genes in the 17-gene signature described by $\mathrm{Ng}$ et al. (Figure 7D) (41).

To understand the effectiveness of ARV-825 in targeting LSC fractions, we performed CyTOF and spanning-tree progression analysis of density-normalized events (SPADE) to study phenotypically defined subpopulations of AML cells and their signaling in primary AML samples treated with ARV-825 in a PDX model. Single-cell proteomics by CyTOF can simultaneously measure the expression of cell-surface and intracellular proteins and can determine protein expression in phenotypically defined rare cell populations. With SPADE 3.0, cell populations from BM were clustered hierarchically according to the expression of surface markers and were displayed in a single minimal spanning tree, where nodes could be annotated for further analysis. LSC populations, defined as $\mathrm{CD} 4^{+} \mathrm{CD} 8^{-} \mathrm{CD}^{-} 0^{-} \mathrm{CD} 45 \mathrm{RA}^{+}$, clustered as a single node in the tree, and the expression of individual surface markers in the SPADE tree of mouse BM cell populations are presented in Figure 7E. In a heatmap of gene expression in LSCs (Figure 7E) and a clustering tree with a single node (highlighted red area in Supplemental Figure 7D), we observed downregulation of Myc activity in LSC nodes in ARV-825-treated mice. Additionally, the proteins associated with Wnt/ $\beta$-catenin (active)/Notch, cell cycle/apoptosis, PI3K/AKT/mTOR, and proliferation pathways were downregulated in the LSC fraction, and inhibition of BRD4, Myc, and HEXIM1 was prominent in the LSC fraction as well (heatmap in Figure 7E and highlighted single nodes of the tree in Supplemental Figure 7, E-I). Importantly, tumor microenvironment-associated molecules (surface CXCR4, CD44, p-FAK, and HIF-1 $\alpha$ ) were downregulated in the LSC compartment, mirroring the vitro data (Figure 7E and Supplemental Figure 7J). Confirming the effect of ARV-825 on LSCs in AML, we found in a PDX model that ARV825 decreased LSC frequencies compared with vehicle treatment (11,789 vs. 2766 in vehicle vs. ARV-825).

\section{Discussion}

Our data highlight the exciting prospect of potent BRD4 degradation in AML by PROTAC and the efficacy of that strategy to simultaneously target LSCs and the leukemia microenvironment. The BET family protein BRD4 has been validated as a druggable transcriptional target in AML models with clinically relevant mutations (isocitrate dehydrogenas [IDH], nucleophosmin 1 [NPM1], and FLT3) and translocations (mixed lineage leukemia [MLL]) $(5,42,43)$. Transcriptional downregulation of oncogenic Myc and antiapoptotic BCL-2 and BCL- ${ }_{\mathrm{XL}}$ has been implicated in the antileukemic activity of BETP inhibitors $(5,44)$. By targeting Myc, BETP degraders can have much broader applicability in Myc-driven hematological malignancies beyond AML, including Burkitt's lymphoma/leukemia, chronic lymphocytic leukemia with Richter's transformation, "double-hit" lymphoma, and others $(9,44)$.

Myc and its downstream targets are critical in AML pathobiology, but direct targeting of Myc has been difficult. BRD4-rich super-enhancer-driven transcription of Myc allows for upstream targeting of Myc. Myc regulates the expression of approximately $15 \%$ of all cellular genes including several antiapoptotic molecules (BCL-2 family proteins) and amino acid transporters (45-47). Analysis of published gene expression data from functionally defined LSCs suggested that Myc targets are highly active in LSC fractions. Sustained inhibition and degradation of BETP, and thereby Myc and its transcriptional network, will have an especially profound impact on immunophenotypically defined AML LSCs, as indicated by our findings. ARV-825, as a sustained degrader of BRD4, led to a more potent downregulation of downstream targets including Myc, BCL-2, BCL- ${ }_{\mathrm{XL}}$, and MCL-1 than was seen with a prototypic BRD4 inhibitor like JQ1. This translates into more effective inhibition of cell proliferation and induction of apoptosis in $\mathrm{CD}_{3} 4^{+}$and $\mathrm{CD} 34^{+} \mathrm{CD} 38^{-}$stem and progenitor populations of primary AML cells, while sparing healthy progenitor cells.

While most work with BRD4 inhibitors has focused on the transcriptional downregulation of cell-intrinsic prosurvival and antiapoptotic proteins, our data, importantly, highlight the modulation of key molecules involved in leukemia and BM microenvironment interactions, namely surface CXCR4 and CD44/CD44v8-10. This is important, because leukemia/BM stroma interactions and the hypoxic microenvironment in the BM can render AML cells resistant to treatment, resulting in a persistence of residual minimal disease. Our data mechanistically link alterations in surface CXCR4 expression at least partly to the transcriptional downregulation of PIM1 kinase downstream of BETP degradation. PIM1 kinasemediated phosphorylation of CXCR4 in the intracellular domain is implicated in surface reexpression of this receptor (34). Ectopic expression of PIM1 in AML cells partially rescued the surface expression of CXCR4 and migration of cells toward the SDF-1 $\alpha$ gradients. G protein-coupled receptor kinase 6- (GRK6-) and calcineurin-mediated phosphorylation of CXCR4 could be additional mechanisms involved in the surface expression of CXCR4 (48). To the best of our knowledge, this is the first report on the role of sustained BETP degradation in the modulation of chemokine receptors and leukemia-microenvironment interactions.

LSCs depend on oxidative phosphorylation and are sensitive to increased ROS levels (49). Apart from suppressing oxidative phosphorylation (Supplemental Figure 8), ARV-825 transcription- 
A Nonleukemic mice ARV-825
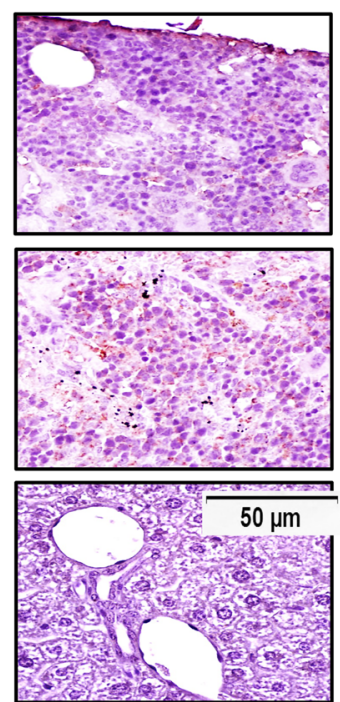
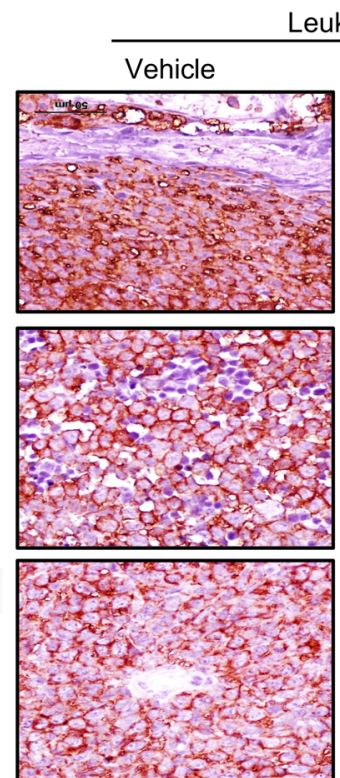

IHC (CD45)
B - Vehicle

-ARV-825

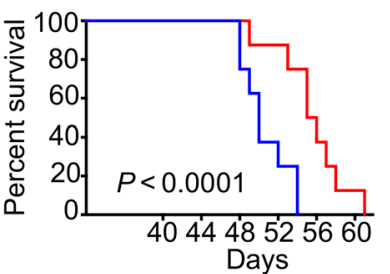

Days
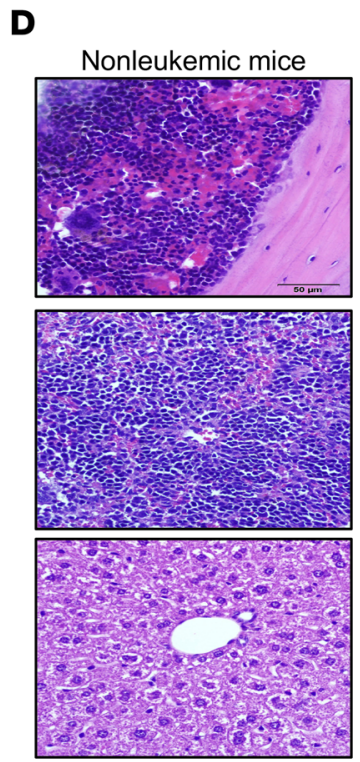

C

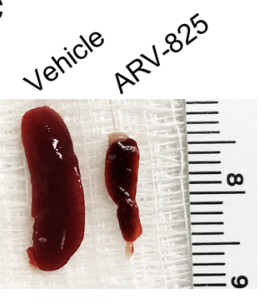

ARV-825
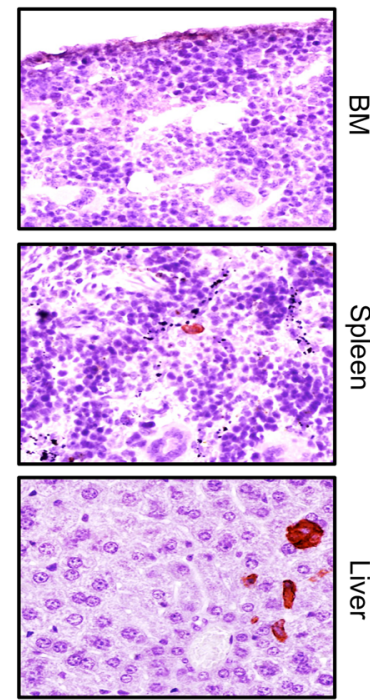

穴
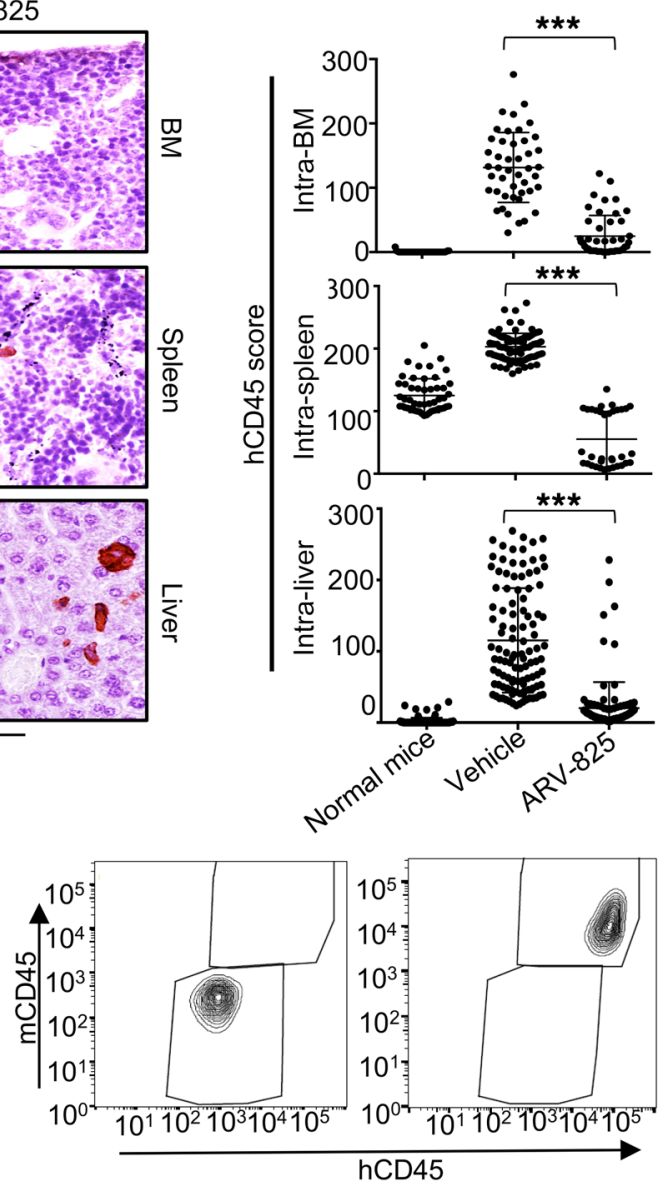

Leukemic mice

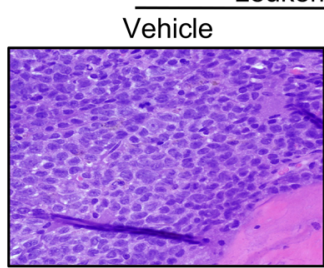
ARV-825

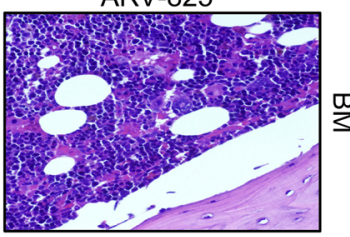

E

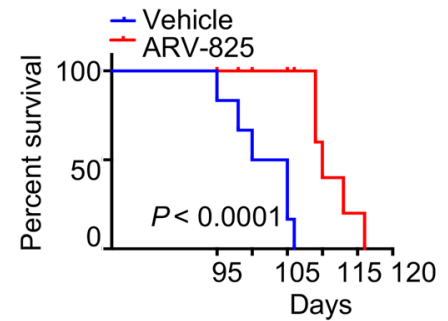


Figure 5. ARV-825 has single-agent antileukemic activity and improves survival of NSG mice engrafted with luciferase-transduced AML cells or AML-PDX cells. Six-week-old NSG mice were injected with luciferaselabeled OCI-AML3 cells $\left(1 \times 10^{6}\right.$ cells) or AML-PDX cells $\left(1 \times 10^{6}\right.$ cells $)$ via the tail vein, and ARV-825 (10 mg/kg) was administered i.p. twice a week $(n=10)$. (A) On day 34, one mouse from each group of mice injected with luciferase-labeled OCI-AML3 cells was sacrificed to assess the infiltration of leukemic cells into other organs, as indicated by the images showing IHC staining for CD45 (original magnification, $\times 40$; scale bar: $50 \mu \mathrm{m}$ ). CD45 $5^{+}$cell infiltration correlated with the response to ARV-825 treatment, as indicated by multispectral analysis of CD $45^{+}$cell quantification. ${ }^{* *} P<0.001$, by standard Student's $t$ test. (B) Kaplan-Meier analysis of overall survival. (C) On day 105 , one mouse from each AML-PDX-engrafted group was sacrificed to assess spleen size, and leukemia burden in the BM was measured by the presence of hCD45+ cells (data were analyzed using Flowjo 10 software). (D) Infiltration of leukemic cells into other organs as indicated by H\&E staining (original magnification, $\times 60$; scale bar: $50 \mu \mathrm{m}$ ). (E) Kaplan-Meier analysis of overall survival probability. mCD45, mouse CD45.

ally downregulates the expression of CD44v8-10. SLC3A11, along with CD44v8-10, forms a heterodimeric amino acid antiporter that maintains cystine levels inside the cell to maintain the cellular redox balance. ARV-825-mediated CD44v8-10 downregulation results in the depletion of intracellular cystine and GSH and increased intracellular ROS (our observations). Gene array data for BM-derived stromal cells showed that ARV-825 also downregulates the stromal counterpart of microenvironment-interacting proteins such as SDF- $1 \alpha$, which probably further impairs stromal support of AML cells.

Studies conducted mainly in the AML, non-Hodgkin lymphoma, and multiple myeloma settings, using BET inhibitors that target BRD4 and acetylated chromatin interaction, have shown promising preclinical results, but the reported clinical responses have been few and short lasting. Lack of sustained inhibition, rebound increases in BRD4 protein levels, non-BRD4-dependent oncogene expression, etc., potentially contribute to this resistance $(15,16$, 18). Additionally, an alternate transcription pathway that drives Myc expression through Wnt/ $\beta$-catenin signaling in LSCs has been recently identified as a mechanism of resistance to prototypic small-molecule BRD4 inhibitors like JQ1 or I-BET compound (15). Our GSEA data show downregulation of the Wnt/ $\beta$-catenin signature with ARV-825, as confirmed by qPCR of selected targets, and suggest that this mechanism of overcoming resistance to BRD4 inhibitors may not be effective against a BRD4 PROTAC. Of note, JQ1-resistant cell lines generated in our laboratory remained sensitive to ARV-825 (data not shown). Indeed, a gene array of primary AML cells suggested that most genes of the 17-gene signature associated with a greater possibility of relapse in AML are downregulated with ARV-825. Of particular importance, the CyTOF data obtained from PDX-bearing mice treated with ARV-825 showed effective modulation of multiple cell-signaling- and microenvironment-related molecules as well as a numerical decrease in phenotypically defined LSC populations, strongly indicating the possibility of LSC elimination with potent BETP degradation.

ARV-825, as a cereblon-based BET PROTAC that recruits the E3 ligase for sustained degradation of BRD4, completely abolished BRD4-dependent oncogenic transcription, showing an enhanced antileukemic effect compared with current BRD4 inhibitors such as JQ1. The improved survival we observed with ARV-825 combined with cytarabine indicates that ARV-825 would be an effective agent to incorporate into future AML treatment regimens.

In summary, our study shows that ARV-825, a BET-targeting PROTAC, has superior antileukemic activity compared with BRD4 inhibitors targeting the interaction of BRD4 with acetylated histone. In addition to downregulating prosurvival and antiapoptotic proteins, ARV-825 modulates tumor-microenvironment interactions and the cellular redox state in AML cells and LSCs (Figure 8). Our findings argue for the further clinical development of ARV-825 to treat AML and potentially other hematological malignancies.

\section{Methods}

AML cell lines and primary samples. Human leukemia cell lines HL60, KBM5, MV4-11, THP-1, and U937 were purchased from the American Type Culture Collection (ATCC). MOLM13, MOLM14, OCI-AML2, and OCI-AML3 were purchased from Deutsche Sammlung von Mikroorganismen und Zellkulturen (DSMZ GmbH). Cells were cultured in RPMI 1640 medium containing $10 \%$ heat-inactivated FBS with penicillin and streptomycin (Sigma-Aldrich) at $37^{\circ} \mathrm{C}$ with $5 \% \mathrm{CO}_{2}$ in a humidified incubator.

HL-60 cell lines with stable overexpression of BCL-2 or BCL- ${ }_{\mathrm{XL}}$ and control lines were provided by Kapil Bhalla (University of Texas MD Anderson Cancer Center).

Peripheral blood mononuclear cells from patients with high blast percentage were cultured in RPMI 1640 medium containing 10\% heat-inactivated FCS supplemented with penicillin and streptomycin at $37^{\circ} \mathrm{C}$ with $5 \% \mathrm{CO}_{2}$ in a humidified incubator. Details of samples are listed in Supplemental Table 5.

For the hypoxia experiments, AML cell lines were cultured in an atmosphere containing $1 \% \mathrm{O}_{2}$ for 14 days to adapt them to hypoxic conditions before performing the experiments using the same culture conditions.

Reagents and antibodies. ARV-825 and JQ1 were obtained from Arvinas Inc. MZ1 was purchased from Cayman Chemical (CAS no. 1797406-69-9) and 2-hydroxypropyl-B-cyclodextrin from Sigma-Aldrich (CAS no. 128446-35-5). Antibodies against BETP (product no. 13440), c-Myc (product no. 5605), cleaved PARP (product no. 9541), p-ERK1/2 (product no. 4370), and PIM1 (product no. 3247) were obtained from Cell Signaling Technology. Other antibodies purchased were: p-CXCR4 (S339) (catalog GTX32281) from GeneTex; p-CXCR4 (S324/S325) (catalog CP4251) from ECM Bioscience; $\beta$-actin (catalog sc-47778), BCL-2 (catalog sc-7382), BCL- ${ }_{\mathrm{xL}}$ (catalog sc-56021), and $\alpha$-tubulin (catalog sc-53646) from Santa Cruz Biotechnology; MCL-1 (catalog BD 559027) from BD Bioscience; and CXCR4 (catalog ab1670), Noxa (catalog ab13654), p- $\gamma \mathrm{H} 2 \mathrm{AX}$ (S139) (catalog ab11174), and H2AX (catalog ab11175) from Abcam.

Apoptosis and viability determination by flow cytometry. AML cells were incubated with ARV-825 or JQ1 for dose- and time-dependent studies. Apoptosis was analyzed using a Gallios Flow Cytometer (Beckman Coulter) following staining with annexin V-FITC (Thermo Fisher Scientific). CountBright Beads (Molecular Probes) and DAPI (Sigma-Aldrich) were used to quantitate the number of viable cells as well as absolute cell numbers. $\mathrm{IC}_{50}$ values were calculated using Calcusyn software (BIOSOFT) on the basis of the number of viable cells, as described previously (50).

Apoptosis of AML stem/progenitor cells was determined as previously described (51). Briefly, AML mononuclear cells were enriched by Ficoll density centrifugation and cultured with increasing concentrations of ARV-825. After 72 hours, AML cells were pelleted via cen- 
A

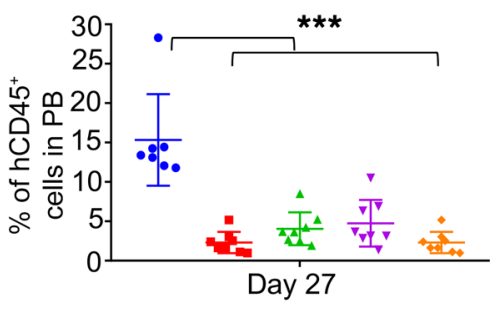

B

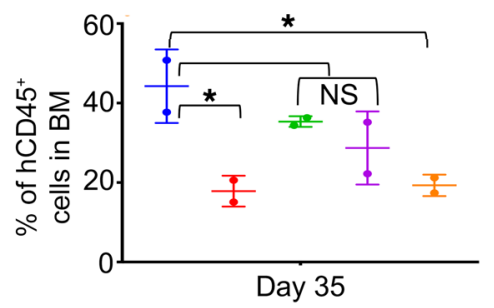

Vehicle $\Delta$ JQ1

ARV-825 $\nabla$ Ara-C ARV-825 + Ara-C

C
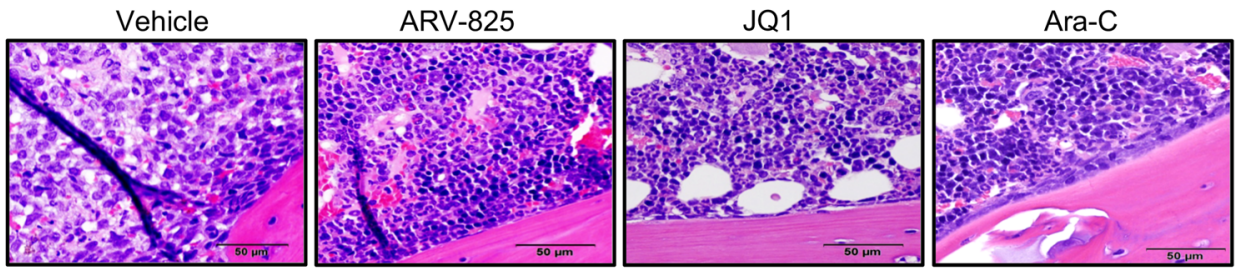

ARV-825 + Ara-C
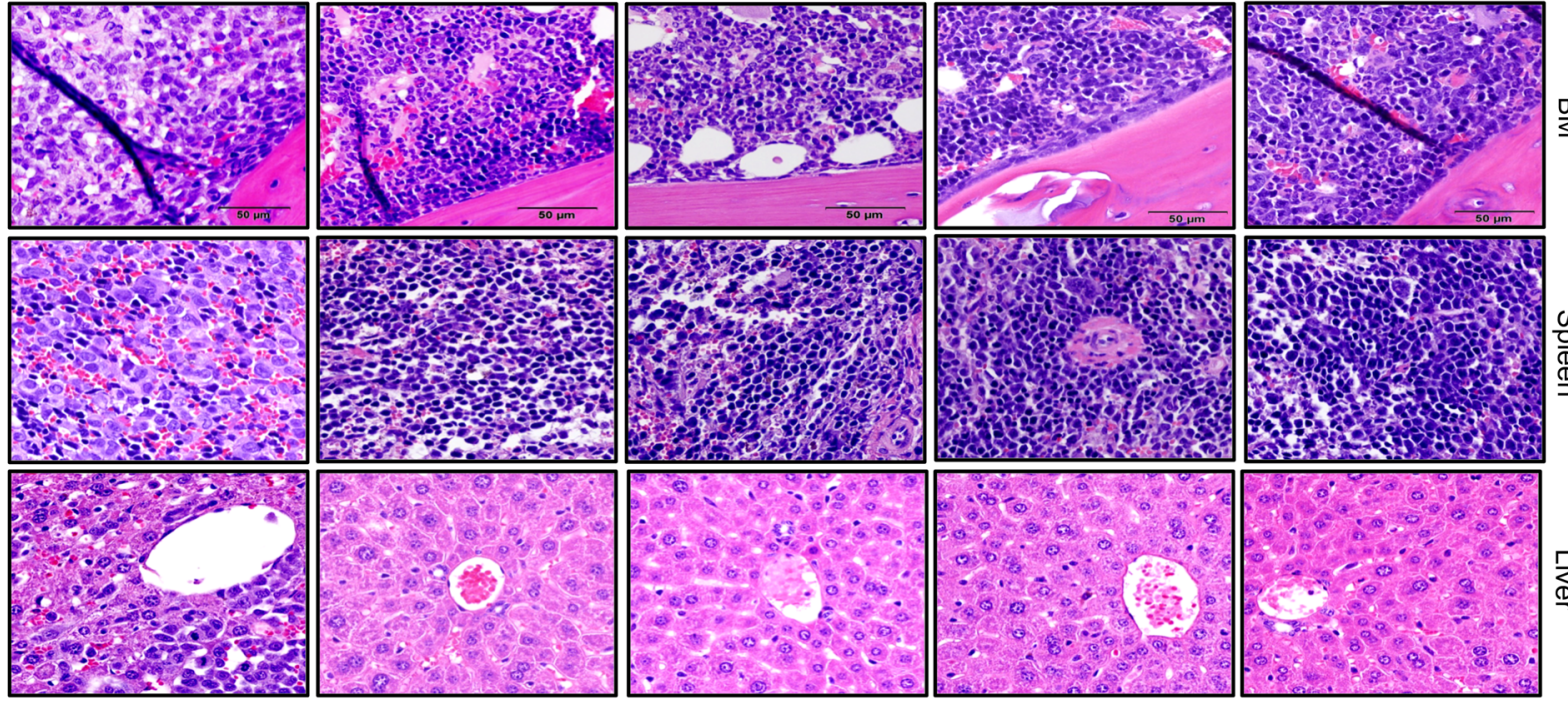

$\stackrel{\text { W }}{3}$
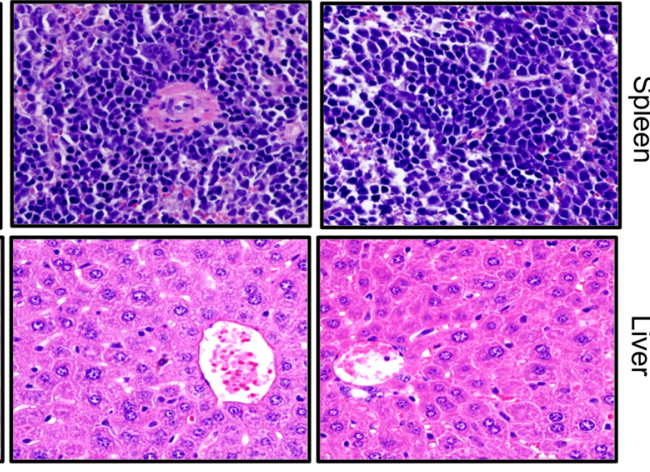

$H \& E$

D

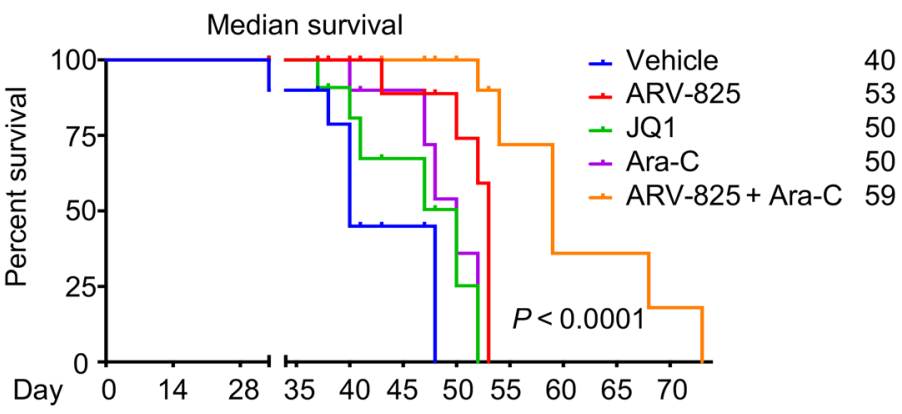

Figure 6. ARV-825 is synergistic with cytarabine, has a more potent antileukemic effect than JQ1, and results in better survival of NSG mice engrafted with luciferase-transduced AML cells. NSG mice were implanted with GFP-luciferase-expressing OCI-AML3 cells and treated with different drugs as indicated $(n=8)$. (A) On day 27, leukemia burden was measured as the percentage of hCD45+ cells in peripheral blood (PB). (B) On day 35 , two mice from each group were sacrificed, and leukemia burden in BM was measured by the percentage of hCD45+ cells (data were analyzed by Flow)o, version 10). (A and B) ${ }^{*} P<0.05$ and ${ }^{* * *} P<0.001$, by standard Student's $t$ test. (C) H\&E-stained images showing infiltration of leukemic cells into other organs. Original magnification, $\times 60$; scale bars: $50 \mu \mathrm{M}$. (D) Kaplan-Meier plot of the in vivo activity of ARV-825 as a single agent, compared with JQ1 (ARV-825 vs. JQ1, $P=0.0126$ ) or combined with cytarabine $(P<0.0001)$.

trifugation, washed twice in annexin-binding buffer $(\mathrm{ABB})$, and stained with $100 \mu \mathrm{l}$ ABB containing a 1:100 dilution of annexin V-APC (cata$\log 550475$ ), and a 1:50 dilution of CD45-APC-Cy7 (catalog 557833), CD34-FITC (catalog 555821), and CD38-PE-Cy7 (catalog 335790) (BD Biosciences) in for 20 minutes at room temperature in the dark. After staining, the cells were washed with $1 \mathrm{ml} \mathrm{ABB}$ containing $0.5 \mu \mathrm{l}$ DAPI $(1 \mathrm{mg} / \mathrm{ml})$. Finally, cells were resuspended in $200 \mu \mathrm{l}$ ABB and analyzed using a Gallios Flow Cytometer (Beckman Coulter).

Surface and intracellular staining and CyTOF analysis. The antibodies used for CyTOF are listed in Supplemental Table 3. AML cells were incubated with ARV-825 for 12 hours and 24 hours. Cells $\left(1 \times 10^{6}\right)$ were subjected to surface and intracellular staining with metal-conjugated antibodies following a previously described method (52). Samples were analyzed using a CyTOF mass cytometer fitted with an AS5 autosampler (DVS Sciences). The bead signature was routinely applied to normalize the CyTOF data before analysis. The data were analyzed by FlowJo software (version 9).

BM cells isolated from mice bearing disseminated AML-PDX on day 95 (vehicle and ARV-825 treated) were subjected to surface and intracellular staining, and data were analyzed by SPADE 3.0. A SPADE tree map was generated using all surface markers of representative LSCs in phenotypically defined myeloid cell populations. The 
boxed annotations were identified by the expression of CD34, CD38, CD90, and CD45RA. The LSC population, as defined by previous studies $\left(\mathrm{CD} 34^{+} \mathrm{CD} 38^{-} \mathrm{CD} 90^{-} \mathrm{CD} 45 \mathrm{RA}^{+}\right)$, was located in the SPADE tree as cluster 1 with surface markers of LSCs. The SPADE tree was illustrated using colors to represent the intensities of different proteins, and GraphPad Prism 10 was used to generate a heatmap on the basis of the percentiles of intensities with respect to the vehicle, while arcsinh-transformed counts for each protein are shown in boxes for CD34 ${ }^{+}$CD $38^{-}{ }^{-}$CD90-CD45RA ${ }^{+}$LSCs.

Cell migration assay. Migration assays were carried out using a Corning HTS 24 Transwell system (Sigma-Aldrich) as described previously (53). Briefly, OCI-AML3 parental/PIM1-overexpressing cells were treated with ARV-825 $(10 \mathrm{nM})$ or plerixafor $(100 \mathrm{nM})$ (positive control). After a 24 -hour incubation, $2 \times 10^{5}$ cells in $200 \mu \mathrm{l}$ serum-free media were seeded onto the insert cup and placed over the receiver well containing serum-free media with SDF-1 (100 ng). Four hours after incubation, cells were collected from the receiver well and counted with trypan blue staining using a Beckman Coulter Vi-CELL counter. Similar experiments were carried out using primary AML cells.

CXCR4 surface staining and flow cytometry. The ARV-825-treated AML cell lines were stained as described previously (21). Briefly, cells were incubated with saturating concentrations of allophycocyaninconjugated anti-CXCR4 monoclonal antibody (12G5; BD Pharmingen, $\mathrm{BD}$ Biosciences) for 30 minutes at room temperature. The appropriate isotype-matched antibody was used as a negative control. The expression of surface CXCR4 was analyzed using a Gallios Flow Cytometer (Beckman Coulter).

Immunofluorescence staining and confocal microscopy. OCI-AML3 cells were treated with vehicle control or ARV-825 and subjected to staining with and without permeabilization, as described previously (54). Briefly, cells were spun onto slides and fixed with $4 \%$ paraformaldehyde for 10 minutes, permeabilized or not with $0.5 \%$ Triton X-100 for 10 minutes, blocked with 5\% BSA in PBS for 1 hour, and then stained at room temperature for 2 hours with anti-CXCR4 antibody (1:100; Abcam). After incubation, cells were washed with PBS and incubated for 30 minutes with Alexa Fluor 594 goat anti-rabbit IgG (1:1000; Invitrogen, Thermo Fisher Scientific). After the final wash with PBS, cells were counterstained with DAPI solution and mounted with mounting medium. Cells were visualized and microphotographed with a FV1000 confocal laser-scanning microscope (Olympus).

Measurement of amino acid uptake and oxygen consumption. OCIAML3 cells were incubated at a density of $5 \times 10^{6}$ cells in $10 \mathrm{ml}$ medium with $10 \mathrm{nM}$ ARV-825 or vehicle control for 0 or 24 hours. After washing twice with PBS, cells were extracted by adding $500 \mu \mathrm{l}$ acetonitrile/water (9:1 ratio) with $1 \%$ formic acid and then disrupted for 2 minutes using a multitube vortexer. Samples were then centrifuged at 20,000 $g$ for 10 minutes, the supernatant was transferred into a new tube and evaporated to dryness, and the samples were reconstituted by adding $200 \mu \mathrm{l}$ acetonitrile/water (9:1) with $1 \%$ formic acid. Samples were analyzed by liquid chromatography-tandem mass spectrometry (LC-MS/MS) with an Agilent 1290 LC system coupled to a 6460 triple quadrupole mass spectrometer at the MD Anderson Proteomics and Metabolomics Facility as described previously $(55,56)$.

Glutathione assay. Cellular GSH levels were measured using a GSH assay kit (Cayman Chemical) according to the manufacturer's instructions. Briefly, cell extracts were sonicated in 0.5× MES buffer (i.e., $50 \mathrm{mM}$ MES or phosphate, $\mathrm{pH}$ 6-7, containing $1 \mathrm{mM}$ EDTA) and deproteinated with metaphosphoric acid. The deproteinated supernatant was collected and then neutralized with triethanolamine. Total GSH levels were detected by measuring the product of glutathionylated 5-5'-dithiobis-2-nitrobenzoic acid with a Victor X3 ultraviolet spectrophotometer (PerkinElmer) at $405 \mathrm{~nm}$. Cellular GSH content was calculated from triplicate measurements using standard curves generated in parallel experiments with standards.

Measurement of total ROS. Total ROS levels of AML cells were measured using an ENZ-51011 Kit (Enzo Life Sciences) according to the manufacturer's protocol. Briefly, $5 \times 10^{5}$ cells were pelleted and labeled with the ROS detection dye (oxidative stress detection reagent, green) for 30 minutes at $37^{\circ} \mathrm{C}$ in the dark. After washing with the buffer, labeled cells were analyzed using a Gallios Flow Cytometer (Beckman Coulter), and the data were assessed using Gallios software (Kaluza). Similar experiments were performed with and without treatment of $N$-actetyl cysteine $(5 \mu \mathrm{M})$.

Generation of PIM1-overexpressing cells. The ORF for human PIM1 and Myc from IMAGE 4591723 and IMAGE 2985844, respectively, was generated by PCR using Q5 High-fidelity Hot Start Polymerase (New England BioLabs). This was cloned with NheI and EcoR1 for PIM1 and NheI and Bam H1 to allow insertion at the Myc sites of a lentiviral vector (pCDH-EF1-MCS-BGH-PGK-GFP-T2A-Puro, System Biosciences). Sanger sequencing verified clones, and the empty vector was used as a negative control. Lentivirus was prepared by transient cotransfection of HEK293T cells (ATCC) with an equimolar mix of transfer vector and packaging plasmids (psPAX2 and pMD2. G, Addgene plasmids 12260 and 12259, respectively) using JetPrime Transfection Reagent as directed by the manufacturer (Polyplus). Lentiviral supernatants were harvested 40-60 hours after transfection and passed through $0.45-\mu \mathrm{m}$ surfactant-free cellulose acetate membranes. AML cell lines were incubated with undiluted viral supernatants overnight at $37^{\circ} \mathrm{C}$ under $5 \% \mathrm{CO}_{2}$. Transfected cells were then washed and selected with puromycin (InvivoGen), starting at $0.5 \mu \mathrm{g} /$ $\mathrm{ml}$. Expression of each transgene was verified by immunoblot analysis.

Immunoblotting. Cells were lysed in RIPA buffer (1\% NP-40, 0.5\% sodium deoxycholate, $0.1 \%$ SDS, $50 \mathrm{mM}$ Tris-Cl [pH 7.5], and $150 \mathrm{mM}$ $\mathrm{NaCl}$ ) in the presence of $1 \times$ protease cocktail inhibitor. Soluble lysates were subjected to SDS-PAGE and transferred onto a PVDF membrane (Bio-Rad). The membranes were probed with specific antibodies. Signals were visualized using the Odyssey Infrared Imaging System (LI-COR Biosciences) and quantitated using Image Studio Lite (LI-COR Biosciences). $\beta$-Actin or $\alpha$-tubulin was used as a loading control.

$q P C R$. RNA was isolated using a PureLink RNA Mini Kit (Ambion, Thermo Fisher Scientific). Concentration was measured with a NanoDrop 2000 UV-Vis Spectrophotometer (Thermo Fisher Scientific), and 500 ng RNA was converted to cDNA using the Verso cDNA Synthesis Kit (Thermo Fisher Scientific). cDNA ( $1 \mu$ l, equivalent to 10 ng starting RNA) was used for each reaction of qPCR in triplicate. qPCR was performed in triplicate using PowerUp SYBR Green Master Mix (Applied Biosystems, Thermo Fisher Scientific) with a 7900HT Fast Real Time-PCR system (Applied Biosystems), as directed by the manufacturer, and subjected to analysis using RQ Manager, version 1.2.1 (Applied Biosystems). Primers were prepared by Integrated DNA Technologies (IDT) and used at a concentration of 500 nM. Primer sequences are listed in Supplemental Table 4.

GEP. OCI-AML3 or primary AML cells or NMSCs treated with ARV-825 were harvested after 24 hours. Total RNA was extracted, 
A

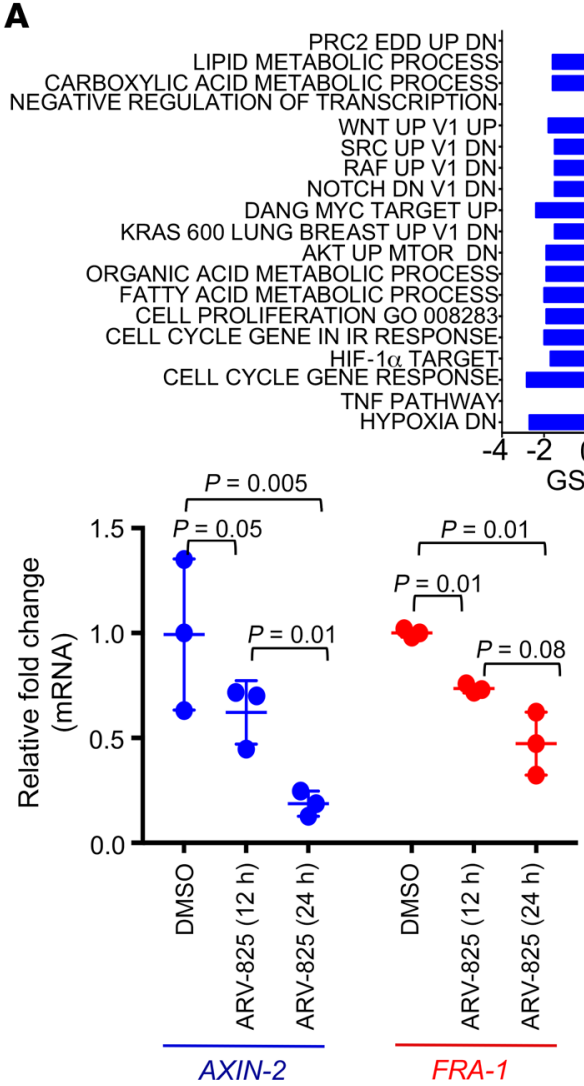

D
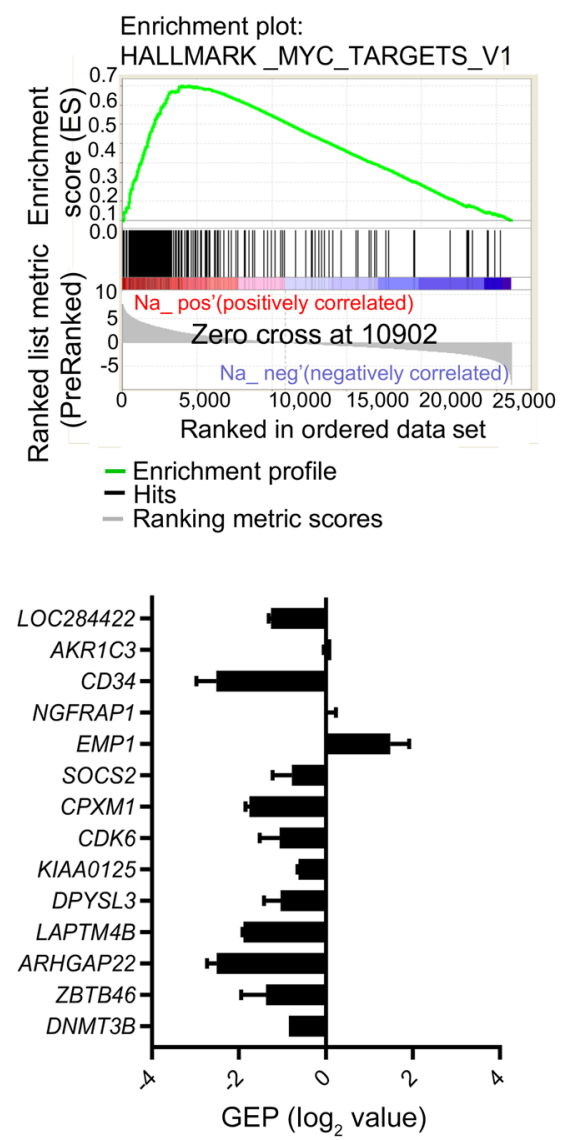

B
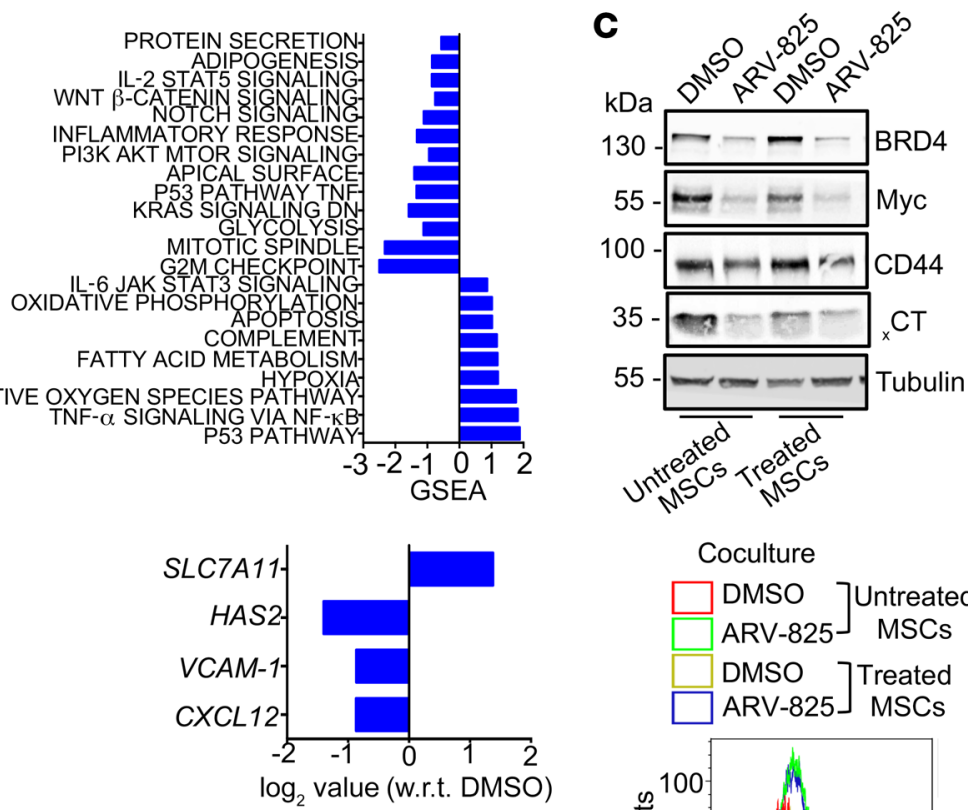

Coculture
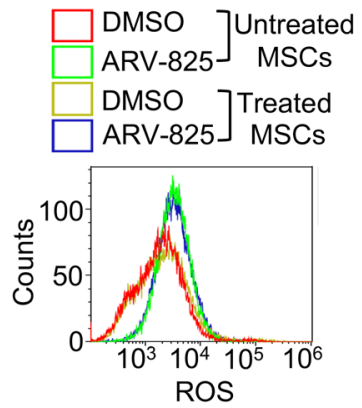

(CD45-gated population)

$\mathbf{E}$
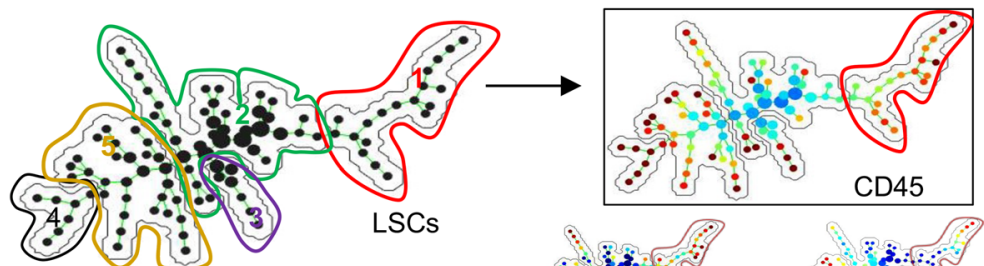

1: CD34 ${ }^{+}$CD $38^{-}{ }^{-C D} 90^{-}$CD45RA ${ }^{+}$(LSCs)

2: CD34-CD38-CD90-CD45RA dim

3: $\mathrm{CD} 34^{-} \mathrm{CD} 38^{+} \mathrm{Cd} 90^{\operatorname{dim}} \mathrm{CD} 45 \mathrm{RA}^{-}$

4: $\mathrm{CD} 34^{\mathrm{dim}} \mathrm{CD} 38^{+} \mathrm{Cd} 90^{+} \mathrm{CF} 45 \mathrm{RA}{ }^{+}$

5: CD34- Cd38 ${ }^{\mathrm{dim}} \mathrm{Cd} 90^{-} \mathrm{CD} 45 \mathrm{RA}^{+}$

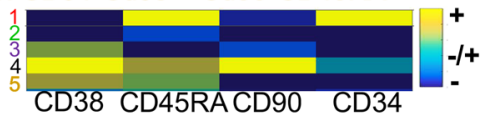

Myc activity

$\overline{\text { Vehicle ARV-825 }}$

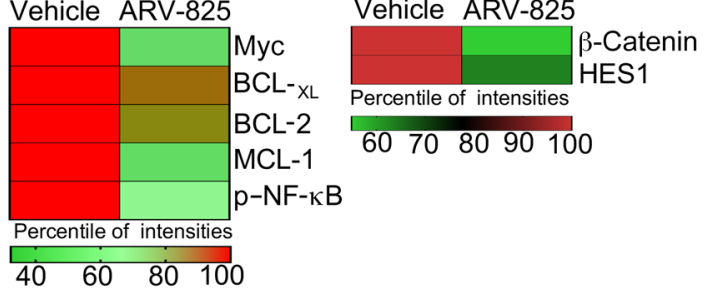

PI3K/AKT/mTOR

Vehicle ARV-825

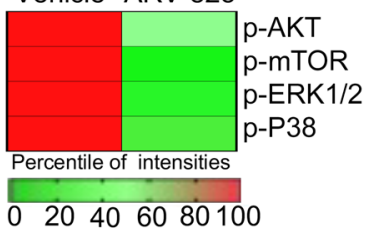

Target inhibition

Vehicle ARV-825

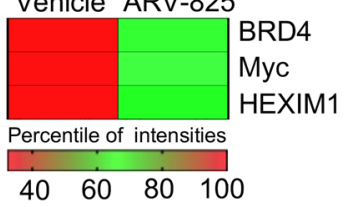

$\begin{array}{llll}40 & 60 & 80 & 100\end{array}$

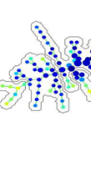

CD34

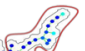

CD90

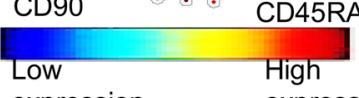

expression

Cell cycle/Apoptosis

Vehicle ARV-825

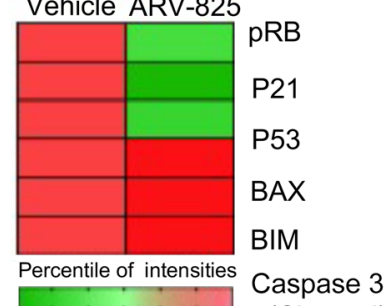

$5060708090100 \quad$ (Cleaved)

Tumor microenvironment

Vehicle ARV-825

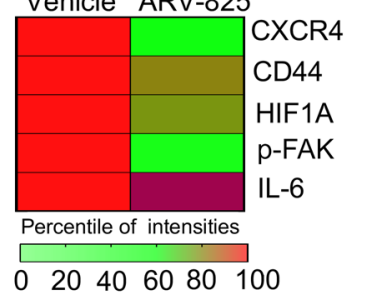


Figure 7. ARV-825 downregulates the Wnt/ $\beta$-catenin pathway, c-Myc transcriptional programs, and other oncogenic pathways and modulates the stromal environment. (A) OCI-AML3 and primary AML cells were treated with ARV-825 (10 $\mathrm{nM})$ for 24 hours. GEP analysis was performed on the isolated RNA $(n=3)$. (A) GSEA was performed on Illumina CEP data and revealed high enrichment (normalized enrichment score [NES] >3) for several gene sets representing downregulation of Myc target genes and Wnt/B catenin pathways, as well as oncogenic pathways and gene sets for cell cycle, hypoxia, metabolism, and Notch). Validation of Wnt/ $\beta$-catenin pathway downregulation was done by qPCR analysis of the pathway targets $A X I N-2$ and $F R A-1$ at 12 and 24 hours ( $n=3$ independent samples). Statistical significance was calculated using Bonferroni's method, and adjusted $P$ values were determined. NMSCs were treated with ARV-825 (25 nM) for 24 hours. (B) Representative GSEA found high enrichment for several gene sets representing Myc target genes and oncogenic pathways, as well as cell-cycle, metabolic, hypoxia, oxidative phosphorylation, and Notch gene sets, and GEP of MSCs showed a significant reduction in surface adhesion and SDF-1 expression. w.r.t., with respect to. (C) MSCs treated with DMSO or ARV-825 ( $25 \mathrm{nM}$ for $24 \mathrm{~h}$ ) cocultured with OCI-AML3 cells treated with DMSO or ARV-825 (10 nM) for 24 hours and subjected to either determination of ROS using an ENZ51011 kit (bottom) or whole-cell lysates from AML cells were subjected to immunoblotting with specific antibodies. Tubulin was used as a loading control. (D) GSE76009 data were curated, and GSEA revealed high enrichment (NES >3) of LSC fractions for several gene sets representing hematopoietic stems cells or LSCs (data not shown) and for this gene set of a subgroup of genes regulated by Myc. Illumina GEP data on primary AML cells treated with ARV- 825 for 24 hours showed a significant reduction in the number of genes of the 17-gene signature associated with stemness in AML. (E) BM cells were collected from vehicle- and ARV-825-treated mice with AML-PDX and subjected to CyTOF, and data were analyzed using SPADE 3.0. The tree was generated according to the expression of CD34, CD38, CD90, and CD45RA (top). Myc activity, Wnt/ $\beta$-catenin/ Notch, cell-cycle/apoptosis, and PI3K/AKT/mTOR pathways, and target inhibition-, and tumor microenvironment-related protein expression levels in BM cells from vehicle- and ARV-825-treated mice were determined and quantified in LSCs (CD34+CD38-CD90-CD45RA $\left.{ }^{+}\right)$as cluster 1 and depicted as a heatmap generated with GraphPad Prism 7 on the basis of the percentiles of intensities with respect to the vehicle (bottom). UP, upregulated; DN, downregulated.

amplified, and labeled by in vitro transcription and then hybridized to Illumina HT12, version 4, human whole-genome arrays as described previously (57). The data were deposited in the NCBI's GEO database (GEO GSE97707).

In vivo model of human $A M L$ and $A M L-P D X$. Twenty male NSG mice ( 6 weeks of age, The Jackson Laboratory) were i.v. injected with luciferase-labeled OCI-AML3 cells $\left(1 \times 10^{6}\right.$ cells $\left./ 100 \mu \mathrm{l}\right)$ and then monitored daily for evidence of leukemia. After 7 days, the mice were chosen randomly for treatment with vehicle or ARV-825 (10 $\mathrm{mg} / \mathrm{kg}$, i.p. twice/week, $n=10$ each) formulated in 5\% ethanol, $20 \%$ solution HS 15, and 75\% D5W (5\% dextrose in water). Bioluminescence imaging was used to monitor tumor burden at different time points. Mice were anesthetized and injected i.p. with firefly luciferase substrate d-luciferin and then imaged noninvasively using the IVIS-200 in vivo imaging system (PerkinElmer). On day 29, peripheral blood was collected via the retro-orbital route and processed to measure leukemia burden (hCD $45^{+}$cells) by flow cytometry as described previously (29). On day 34 , one mouse from each group was sacrificed by $\mathrm{CO}_{2}$ asphyxiation and cervical dislocation, and then the femur, spleen, and liver were collected for H\&E and immunohistochemical staining. Immunostained specimens were assessed with multispectral immunohistochemical analysis using the Vectra 3.0 Automated Quantitative Pathology Imaging System, which provided simultaneous detection and quantitation of hCD45 staining. Survival of the mice was also followed, and survival data are presented in a Kaplan-Meier survival plot.

Similarly, for the AML-PDX model, 14 male NSG mice (6 weeks of age, The Jackson Laboratory) were i.v. injected with AML-PDX $\left(1.5 \times 10^{6}\right.$ cells $\left./ 100 \mu \mathrm{l}\right)$. After 21 days, peripheral blood was collected via the retro-orbital route and processed to measure $\mathrm{hCD} 45^{+}$cells by flow cytometry to confirm the establishment of leukemia. On day 22 , the mice were grouped for treatment with vehicle or ARV-825 (10 $\mathrm{mg} / \mathrm{kg}$, i.p. twice/week, $n=6$ each). On day 75 , peripheral blood was collected through the retro-orbital route and processed to measure leukemia burden ( $\mathrm{hCD} 45^{+}$cells) as well as to measure target inhibition and surface CXCR4 and CD44 expression by flow cytometry as described previously (29). On day 95, one mouse from each group was sacrificed to isolate spleen and bone marrow. BM processed for proteomic analysis by CyTOF. Additionally, on day 106, one mouse from each group was sacrificed, and the femur, spleen, and liver were collected for H\&E and immunohistochemical staining. The rest of mice were followed for survival.

For comparative and combination studies, OCI-AML3-GFP-Luc leukemic cells were transplanted as mentioned above into 40 NSG mice. On day 11, the mice were chosen randomly for treatment with vehicle or ARV-825 (10 mg/kg), JQ1 (50 mg/ kg, formulated in 5:95 DMSO: 10\% 2-hydroxypropyl-B-cyclodextrin, Sigma-Aldrich), cytarabine $(50 \mathrm{mg} / \mathrm{kg})$, or a combination of ARV-825 and cytarabine $(n=$ 8) i.p. twice a week. Bioluminescence imaging was used to monitor tumor burden at different time points as mentioned above. On day 27, peripheral blood was processed to measure leukemia burden (hCD $45^{+}$ cells) by flow cytometry as described above. On day 34 , one mouse from each group was sacrificed by $\mathrm{CO}_{2}$ asphyxiation and cervical dislocation and then the femur, spleen, and liver were collected for $\mathrm{H} \& \mathrm{E}$ staining and hCD45 flow cytometric analysis of BM. The rest of the mice were followed for survival.

GSEA. GSEA was performed on Illumina GEP data (GEO GSE6009) from the study by $\mathrm{Ng}$ et al. (41). Jean Wang provided indicators of paired samples, i.e., ones for which 1 or more LSC fractions and 1 or more non-LSC fractions were from the same patient. For each patient with paired samples, data for the LSC and non-LSC fractions were averaged separately, and then gene probes were ranked according to the $Z$ scores of a paired $t$ test, with averaging of the $Z$ scores for different probes for the same gene.

Statistics. All data are expressed as the mean \pm SD and are representative of triplicate samples. Statistics were generated using GraphPad Prism 7 software. Kaplan-Meier survival curves were generated to compare the in vivo survival data using the log-rank test. Normally distributed groups were compared by a 2-tailed Student's $t$ test. All statistical evaluations were 2 sided. $P$ values of 0.05 or less were considered statistically significant for single comparisons. For multiple comparisons, Bonferroni's correction was used to adjust for the inflated type I error.

Study approval. Primary AML specimens were collected from patients with their written informed consent, in accordance with the institutional guidelines of the University of Texas MD Anderson Cancer Center and declaration of Helsinki principles. All animal studies were approved by the IACUC of the University of Texas MD Anderson Cancer Center (protocol 00001516-RNO0). 


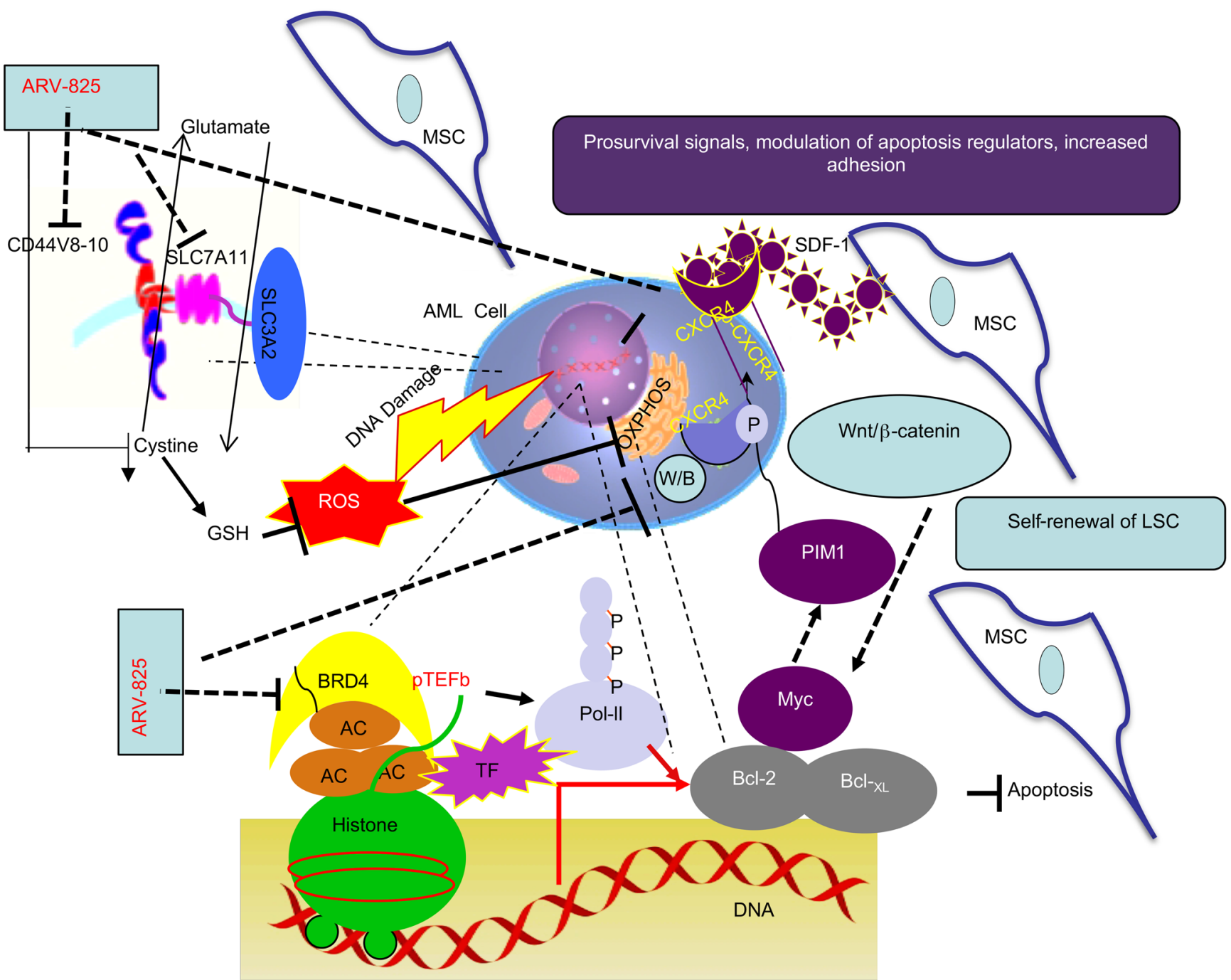

Figure 8. Simultaneous targeting of leukemia-intrinsic biology and leukemia-microenvironment interaction will be critical for the successful treatment of AML. ARV-825, a cereblon-based BET-targeting PROTAC, causes sustained BRD4 degradation accompanied by downregulation of targets such as Myc and BCL-2 family molecules, resulting in increased apoptosis and DNA damage. While modulation of chemokine receptor (inhibition of surface expression of CXCR4)/ adhesion molecules (transcriptional downregulation of CD44) and Wnt/ $\beta$-catenin signaling results inactivation of prosurvival signals, modulation of apoptosis regulators, decreased adhesion and impair of self-renewal of leukemic stem cells and persistence residual clones. As a functional correlative of CD44v8-10 downregulation, a reduction in cysteine uptake and cellular GSH levels results in increased ROS generation and mitochondrial metabolic inhibition (OXPHOS). Hence, the antileukemic activity of BET protein degradation by ARV-825 is associated with modulation of chemokine receptors, cell adhesion, and metabolic targets.

\section{Author contributions}

SP, GB, and MA, conceived and designed the study. SP, GB, MA, $\mathrm{HM}$, and PLL contributed to the study development and methodology. SP, HM, SB, NB, PLL, and RED handled data acquisition. SP, GB, MA, PLL, VR, SB, and MK, wrote, reviewed, and revised the manuscript. TM, GB, and MA, and MK provided administrative, technical, and material support. ZW contributed to GEP. YQ provided funding. CMC, JI, MJY, and HK reviewed the manuscript.

\section{Acknowledgments}

We wish to thank Lina Han, Zhihong Zeng, and Qi Zhang for AMLPDX assistance and CyTOF data analysis; Jared K. Burks and Duncan H. Mak for flow cytometric analysis and cellular imaging; Qiao Wei for statistical analysis and correction (all from the University of Texas MD Andersen Cancer Center); and members of the Konople- va and Andreeff laboratory at the University of Texas MD Anderson Cancer Center for helpful comments and suggestions. We thank the members of the Flow Cytometry and Cellular Imaging Core Facility at the University of Texas for technical and material support and give special thanks to Numsen M. Hail Jr. for editing the manuscript. This work was supported in part by funding from the NIH (CA016672); the Cancer Prevention Research Institute of Texas (RP121010); the Paul and Mary Haas Chair in Genetics (to MA); and Arvinas Inc.

Address correspondence to: Gautam Borthakur or Michael Andreeff, UT MD Anderson Cancer Center, 1515 Holcombe Boulevard. Unit Number: 428, Department of Leukemia -FC4.2006, Houston, Texas 77030, USA. Phone: 713.563.1586; Email: gborthak@mdanderson.org (GB). Phone: 713.792.7261; Email: mandreef@mdanderson.org (MA). 
1. De Kouchkovsky I, Abdul-Hay M. 'Acute myeloid leukemia: a comprehensive review and 2016 update'. Blood Cancer J. 2016;6(7):e441.

2. Chen J, Odenike O, Rowley JD. Leukaemogenesis: more than mutant genes. Nat Rev Cancer. 2010;10(1):23-36.

3. Dawson MA, Kouzarides T, Huntly BJ. Targeting epigenetic readers in cancer. $N$ Engl J Med 2012;367(7):647-657.

4. Passegué E, Jamieson CH, Ailles LE, Weissman IL. Normal and leukemic hematopoiesis: are leukemias a stem cell disorder or a reacquisition of stem cell characteristics? Proc Natl Acad Sci U S A. 2003;100(Suppl 1):11842-11849.

5. Zuber J, et al. RNAi screen identifies Brd4 as a therapeutic target in acute myeloid leukaemia. Nature. 2011;478(7370):524-528.

6. Tzelepis K, et al. A CRISPR dropout screen identifies genetic vulnerabilities and therapeutic targets in acute myeloid leukemia. Cell Rep. 2016;17(4):1193-1205.

7. Filippakopoulos P, Knapp S. Targeting bromodomains: epigenetic readers of lysine acetylation. Nat Rev Drug Discov. 2014;13(5):337-356.

8. Asangani IA, et al. Therapeutic targeting of BET bromodomain proteins in castrationresistant prostate cancer. Nature. 2014;510(7504):278-282.

9. Filippakopoulos P, et al. Selective inhibition of BET bromodomains. Nature. 2010;468(7327):1067-1073.

10. Shi J, et al. Role of SWI/SNF in acute leukemia maintenance and enhancer-mediated Myc regulation. Genes Dev. 2013;27(24):2648-2662.

11. Delmore JE, et al. BET bromodomain inhibition as a therapeutic strategy to target c-Myc. Cell. 2011;146(6):904-917.

12. Dawson MA, et al. Inhibition of BET recruitment to chromatin as an effective treatment for MLL-fusion leukaemia. Nature. 2011;478(7370):529-533.

13. Jung $\mathrm{M}$, et al. Affinity map of bromodomain protein 4 (BRD4) interactions with the histone $\mathrm{H} 4$ tail and the small molecule inhibitor JQ1. J Biol Chem. 2014;289(13):9304-9319.

14. Bolden JE, et al. Inducible in vivo silencing of Brd4 identifies potential toxicities of sustained BET protein inhibition. Cell Rep. 2014;8(6):1919-1929.

15. Fong CY, et al. BET inhibitor resistance emerges from leukaemia stem cells. Nature. 2015;525(7570):538-542.

16. Lu J, et al. Hijacking the E3 ubiquitin ligase cereblon to efficiently target BRD4. Chem Biol. 2015;22(6):755-763.

17. Zengerle M, Chan KH, Ciulli A. Selective small molecule induced degradation of the BET bromodomain protein BRD4. ACS Chem Biol. 2015;10(8):1770-1777.

18. Lu H, et al. Compensatory induction of MYC expression by sustained CDK9 inhibition via a BRD4-dependent mechanism. Elife. 2015;4:e06535.

19. Saenz DT, et al. Novel BET protein proteolysis-targeting chimera exerts superior lethal activity than bromodomain inhibitor (BETi) against post-myeloproliferative neo- plasm secondary (s) AML cells. Leukemia. 2017;31(9):1951-1961.

20. Nervi B, et al. Chemosensitization of acute myeloid leukemia (AML) following mobilization by the CXCR4 antagonist AMD3100. Blood. 2009;113(24):6206-6214.

21. Zeng Z, et al. Targeting the leukemia microenvironment by CXCR4 inhibition overcomes resistance to kinase inhibitors and chemotherapy in AML. Blood. 2009;113(24):6215-6224.

22. Bjorklund CC, et al. Evidence of a role for CD 44 and cell adhesion in mediating resistance to lenalidomide in multiple myeloma: therapeutic implications. Leukemia. 2014;28(2):373-383.

23. Jin L, Hope KJ, Zhai Q, Smadja-Joffe F, Dick JE. Targeting of CD44 eradicates human acute myeloid leukemic stem cells. Nat Med. 2006;12(10):1167-1174.

24. Zöller M. CD44, hyaluronan, the hematopoietic stem cell, and leukemia-initiating cells. Front Immunol. 2015;6:235.

25. Quéré R, et al. High levels of the adhesion molecule CD44 on leukemic cells generate acute myeloid leukemia relapse after withdrawal of the initial transforming event. Leukemia. 2011;25(3):515-526.

26. Nagano O, Okazaki S, Saya H. Redox regulation in stem-like cancer cells by CD44 variant isoforms. Oncogene. 2013;32(44):5191-5198.

27. Ishimoto $\mathrm{T}$, et al. $\mathrm{CD} 44$ variant regulates redox status in cancer cells by stabilizing the $\mathrm{xCT}$ subunit of system $\mathrm{xc}(-)$ and thereby promotes tumor growth. Cancer Cell. 2011;19(3):387-400.

28. Lagadinou ED, et al. BCL-2 inhibition targets oxidative phosphorylation and selectively eradicates quiescent human leukemia stem cells. Cell Stem Cell. 2013;12(3):329-341.

29. Piya S, et al. Atg7 suppression enhances chemotherapeutic agent sensitivity and overcomes stroma-mediated chemoresistance in acute myeloid leukemia. Blood. 2016;128(9):1260-1269.

30. Tabe Y, Konopleva M. Advances in understanding the leukaemia microenvironment. Br J Haematol. 2014;164(6):767-778.

31. Zhou HS, Carter BZ, Andreeff M. Bone marrow niche-mediated survival of leukemia stem cells in acute myeloid leukemia: Yin and Yang. Cancer Biol Med. 2016;13(2):248-259.

32. Domanska UM, et al. A review on CXCR4/ CXCL12 axis in oncology: no place to hide. Eur J Cancer. 2013;49(1):219-230.

33. Busillo JM, Benovic JL. Regulation of CXCR4 signaling. Biochim Biophys Acta. 2007;1768(4):952-963.

34. Brault L, Rovó A, Decker S, Dierks C, Tzankov A, Schwaller J. CXCR4-SERINE339 regulates cellular adhesion, retention and mobilization, and is a marker for poor prognosis in acute myeloid leukemia. Leukemia. 2014;28(3):566-576.

35. Grundler R, et al. Dissection of PIM serine/ threonine kinases in FLT3-ITD-induced leukemogenesis reveals PIM1 as regulator of CXCL12-CXCR4-mediated homing and migration. J Exp Med. 2009;206(9):1957-1970.

36. Konoplev S, et al. CXC chemokine receptor
4 expression, CXC chemokine receptor 4 activation, and wild-type nucleophosmin are independently associated with unfavorable prognosis in patients with acute myeloid leukemia. Clin Lymphoma Myeloma Leuk. 2013;13(6):686-692.

37. Winter GE, et al. DRUG DEVELOPMENT. Phthalimide conjugation as a strategy for in vivo target protein degradation. Science. 2015;348(6241):1376-1381.

38. Bhutia YD, Babu E, Ramachandran S, Ganapathy V. Amino acid transporters in cancer and their relevance to "glutamine addiction": novel targets for the design of a new class of anticancer drugs. Cancer Res. 2015;75(9):1782-1788.

39. Gorrini C, Harris IS, Mak TW. Modulation of oxidative stress as an anticancer strategy. Nat Rev Drug Discov. 2013;12(12):931-947.

40. Zhang W, et al. Stromal control of cystine metabolism promotes cancer cell survival in chronic lymphocytic leukaemia. Nat Cell Biol. 2012;14(3):276-286.

41. Ng SW, et al. A 17-gene stemness score for rapid determination of risk in acute leukaemia. Nature. 2016;540(7633):433-437.

42. Chen C, et al. Cancer-associated IDH2 mutants drive an acute myeloid leukemia that is susceptible to Brd4 inhibition. Genes Dev. 2013;27(18):1974-1985.

43. Fiskus $\mathrm{W}$, et al. BET protein antagonist JQ1 is synergistically lethal with FLT3 tyrosine kinase inhibitor (TKI) and overcomes resistance to FLT3-TKI in AML cells expressing FLT-ITD. Mol Cancer Ther. 2014;13(10):2315-2327.

44. Mertz JA, et al. Targeting MYC dependence in cancer by inhibiting BET bromodomains. Proc Natl Acad Sci U S A. 2011;108(40):16669-16674.

45. Li Z, Van Calcar S, Qu C, Cavenee WK, Zhang $\mathrm{MQ}$, Ren B. A global transcriptional regulatory role for c-Myc in Burkitt's lymphoma cells. Proc Natl Acad Sci U S A. 2003;100(14):8164-8169.

46. Hoffman B, Liebermann DA. Apoptotic signaling by c-MYC. Oncogene. 2008;27(50):6462-6472.

47. Hayashi K, Jutabha P, Endou H, Anzai N. c-Myc is crucial for the expression of LAT1 in MIA Paca-2 human pancreatic cancer cells. Oncol Rep. 2012;28(3):862-866.

48. Busillo JM, Armando S, Sengupta R, Meucci O, Bouvier M, Benovic JL. Site-specific phosphorylation of CXCR4 is dynamically regulated by multiple kinases and results in differential modulation of CXCR4 signaling. J Biol Chem. 2010;285(10):7805-7817.

49. Ito K, Suda T. Metabolic requirements for the maintenance of self-renewing stem cells. Nat Rev Mol Cell Biol. 2014;15(4):243-256.

50. Han L, et al. Antileukemia efficacy and mechanisms of action of SL-101, a novel anti-CD123 antibody conjugate, in acute myeloid leukemia. Clin Cancer Res. 2017;23(13):3385-3395.

51. Carter BZ, et al. Synergistic targeting of AML stem/progenitor cells with IAP antagonist birinapant and demethylating agents. J Natl Cancer Inst. 2014;106(2):djt440.

52. Han L, et al. Single-cell mass cytometry reveals intracellular survival/proliferative signaling in FLT3-ITD-mutated AML stem/progenitor 
cells. Cytometry A. 2015;87(4):346-356.

53. Tabe $\mathrm{Y}$, et al. Role of stromal microenvironment in nonpharmacological resistance of CML to imatinib through Lyn/CXCR4 interactions in lipid rafts. Leukemia. 2012;26(5):883-892.

54. Chen Y, Jacamo R, Konopleva M, Garzon R, Croce C, Andreeff M. CXCR4 downregulation of let-7a drives chemoresistance in acute myeloid leukemia. J Clin Invest. 2013;123(6):2395-2407.

55. Purwaha P, Lorenzi PL, Silva LP, Hawke DH, Weinstein JN. Targeted metabolomic analysis of amino acid response to $L$-asparaginase in adherent cells. Metabolomics. 2014;10(5):909-919.

56. Silva LP, Lorenzi PL, Purwaha P, Yong V, Hawke DH, Weinstein JN. Measurement of DNA concentration as a normalization strategy for metabolomic data from adherent cell lines. Anal Chem. 2013;85(20):9536-9542.

57. Battula VL, et al. Connective tissue growth factor regulates adipocyte differentiation of mesenchymal stromal cells and facilitates leukemia bone marrow engraftment. Blood. 2013;122(3):357-366. 